\title{
Design of TTF-Based Phosphazenes Combining a Good Electron-Donor Capacity and Possible Inclusion Adduct Formation (Part II)
}

Godefroid Gahungu, Bin Zhang, Jingping Zhang.

\section{Supporting materials}

\section{Index}

1. Cartesian coordinates of the relevant structures.......................Pages 2-23

2. Figures (S1-S4) not given in the main text.........................Pages 24-25

3. Tables (S1-S4) listing the numerical results not given in the text........Pages 26-31 


\section{Cartesian coordinates of the relevant structures}

\section{TTF}

$\begin{array}{crrr}\text { C } & 0.000000 & 0.000000 & 0.000000 \\ \text { C } & 0.000000 & 0.000000 & 1.348578 \\ \text { S } & 1.487091 & 0.000000 & 2.311648 \\ \text { S } & -1.487091 & 0.000000 & -0.963070 \\ \mathrm{~S} & -1.487126 & 0.000000 & 2.311536 \\ \mathrm{~S} & 1.487126 & 0.000000 & -0.962958 \\ \mathrm{C} & 0.667536 & 0.000129 & 3.855047 \\ \mathrm{C} & -0.667536 & 0.000129 & -2.506469 \\ \mathrm{C} & -0.667706 & 0.000111 & 3.855013 \\ \mathrm{C} & 0.667706 & 0.000111 & -2.506435 \\ \mathrm{H} & 1.284915 & 0.000159 & 4.745140 \\ \mathrm{H} & -1.284915 & 0.000159 & -3.396562 \\ \mathrm{H} & -1.285183 & 0.000184 & 4.745037 \\ \mathrm{H} & 1.285183 & 0.000184 & -3.396459\end{array}$

\section{TMTSF}

$\begin{array}{rrrr}\mathrm{C} & 0.000000 & 0.000000 & 0.000000 \\ \mathrm{C} & 0.000000 & 0.000000 & 1.341824 \\ \mathrm{Se} & 1.569862 & 0.000000 & 2.383217 \\ \mathrm{Se} & -1.569757 & 0.018140 & -1.041393 \\ \mathrm{C} & 0.666587 & -0.596968 & 3.946534 \\ \mathrm{C} & -0.673441 & -0.589226 & -2.604710 \\ \mathrm{C} & -0.673441 & -0.589226 & 3.946534 \\ \mathrm{C} & 0.666587 & -0.596968 & -2.604710 \\ \mathrm{Se} & -1.569757 & 0.018140 & 2.383218 \\ \mathrm{Se} & 1.569862 & 0.000000 & -1.041394 \\ \mathrm{C} & 1.574419 & -1.008344 & 5.060741 \\ \mathrm{C} & -1.585966 & -0.990084 & -3.718917 \\ \mathrm{H} & 2.281319 & -0.205105 & 5.300360 \\ \mathrm{H} & -2.283537 & -0.178731 & -3.958536 \\ \mathrm{H} & 1.021947 & -1.248480 & 5.971177 \\ \mathrm{H} & -1.036305 & -1.236588 & -4.629353 \\ \mathrm{H} & 2.163551 & -1.887607 & 4.777576 \\ \mathrm{H} & -2.185218 & -1.862481 & -3.435752 \\ \mathrm{C} & -1.585966 & -0.990083 & 5.060741 \\ \mathrm{C} & 1.574420 & -1.008343 & -3.718917 \\ \mathrm{H} & -2.283535 & -0.178728 & 5.300363 \\ \mathrm{H} & 2.281317 & -0.205102 & -3.958539 \\ \mathrm{H} & -1.036304 & -1.236590 & 5.971176 \\ \mathrm{H} & 1.021946 & -1.248482 & -4.629352 \\ \mathrm{H} & -2.185221 & -1.862478 & 4.777575 \\ \mathrm{H} & 2.163554 & -1.887604 & -3.435751\end{array}$




\section{BEDT-TTF (ET)}

$\begin{array}{lrrr}\text { C } & 0.000000 & 0.000000 & 0.000000 \\ \text { C } & 0.000000 & 0.000000 & 1.349238 \\ \text { S } & 1.477648 & 0.000000 & -0.965978 \\ \text { S } & -1.475645 & -0.076910 & 2.315215 \\ \mathrm{C} & 0.670854 & 0.637878 & -2.403687 \\ \mathrm{C} & -0.703145 & 0.602096 & 3.752925 \\ \mathrm{C} & -0.677325 & 0.609513 & -2.404241 \\ \mathrm{C} & 0.644682 & 0.643940 & 3.753479 \\ \mathrm{~S} & -1.467412 & -0.066908 & -0.976599 \\ \mathrm{~S} & 1.468906 & 0.009560 & 2.325837 \\ \mathrm{~S} & 1.724526 & 1.188695 & -3.686162 \\ \mathrm{~S} & -1.784059 & 1.097324 & 5.035400 \\ \mathrm{C} & 0.756580 & 0.860640 & -5.218205 \\ \mathrm{C} & -0.800350 & 0.820094 & 6.567443 \\ \mathrm{C} & -0.564362 & 0.149406 & -5.015269 \\ \mathrm{C} & 0.555820 & 0.178578 & 6.364507 \\ \mathrm{~S} & -1.645951 & 0.988299 & -3.809939 \\ \mathrm{~S} & 1.592280 & 1.072630 & 5.159176 \\ \mathrm{H} & 0.592444 & 1.831928 & -5.689787 \\ \mathrm{H} & -0.686990 & 1.798609 & 7.039025 \\ \mathrm{H} & 1.401798 & 0.258721 & -5.866924 \\ \mathrm{H} & -1.413364 & 0.185408 & 7.216162 \\ \mathrm{H} & -1.111488 & 0.135099 & -5.963057 \\ \mathrm{H} & 1.102950 & 0.192768 & 7.312295 \\ \mathrm{H} & -0.423778 & -0.881649 & -4.680944 \\ \mathrm{H} & 0.469093 & -0.858397 & 6.030182 \\ & & & \\ & & & \end{array}$

\section{BETS}

$\begin{array}{rrrr}\text { C } & 0.000000 & 0.000000 & 0.000000 \\ \text { C } & 0.000000 & 0.000000 & 1.342119 \\ \text { Se } & 1.577585 & 0.000000 & -1.035470 \\ \text { Se } & -1.575491 & -0.081253 & 2.377589 \\ \mathrm{C} & 0.677870 & 0.849161 & -2.484635 \\ \mathrm{C} & -0.720706 & 0.813121 & 3.826754 \\ \mathrm{C} & -0.667075 & 0.816025 & -2.490798 \\ \mathrm{C} & 0.624161 & 0.849300 & 3.832917 \\ \mathrm{Se} & -1.558751 & -0.058553 & -1.058532 \\ \mathrm{Se} & 1.559698 & 0.021807 & 2.400651 \\ \mathrm{~S} & 1.753320 & 1.557201 & -3.666905 \\ \mathrm{~S} & -1.831196 & 1.464830 & 5.009024 \\ \mathrm{C} & 0.740967 & 1.644184 & -5.196265 \\ \mathrm{C} & -0.824667 & 1.603838 & 6.538384\end{array}$




$\begin{array}{lrrr}\mathrm{C} & -0.545679 & 0.847261 & -5.157873 \\ \mathrm{C} & 0.501316 & 0.874241 & 6.499992 \\ \mathrm{~S} & -1.656519 & 1.381065 & -3.816858 \\ \mathrm{~S} & 1.583189 & 1.464550 & 5.158977 \\ \mathrm{H} & 1.386184 & 1.271215 & -5.998972 \\ \mathrm{H} & -1.449817 & 1.198133 & 7.341091 \\ \mathrm{H} & 0.523736 & 2.698019 & -5.383167 \\ \mathrm{H} & -0.662002 & 2.667463 & 6.725286 \\ \mathrm{H} & -0.357590 & -0.223449 & -5.041287 \\ \mathrm{H} & 0.368624 & -0.204735 & 6.383406 \\ \mathrm{H} & -1.098183 & 1.006683 & -6.088645 \\ \mathrm{H} & 1.044876 & 1.061908 & 7.430764\end{array}$

\section{BEDO- TTF (BO)}

$\begin{array}{lrrr}\mathrm{C} & -0.001812 & -0.653510 & -0.022324 \\ \mathrm{C} & 0.003340 & -0.653503 & 1.327624 \\ \mathrm{~S} & 1.491909 & -0.678696 & -0.972147 \\ \mathrm{~S} & -1.490317 & -0.682184 & 2.277447 \\ \mathrm{C} & 0.663166 & -0.207073 & -2.452322 \\ \mathrm{C} & -0.662680 & -0.208624 & 3.757622 \\ \mathrm{C} & -0.677151 & -0.203474 & -2.448054 \\ \mathrm{C} & 0.677625 & -0.201889 & 3.753354 \\ \mathrm{~S} & -1.499587 & -0.672604 & -0.964344 \\ \mathrm{~S} & 1.501156 & -0.669095 & 2.269644 \\ \mathrm{O} & 1.435619 & 0.090552 & -3.529454 \\ \mathrm{O} & -1.435827 & 0.087194 & 4.834754 \\ \mathrm{C} & 0.648196 & 0.684923 & -4.555914 \\ \mathrm{C} & -0.649796 & 0.683405 & 5.861214 \\ \mathrm{C} & -0.669234 & -0.056976 & -4.720197 \\ \mathrm{C} & 0.669365 & -0.055411 & 6.025497 \\ \mathrm{O} & -1.450638 & 0.054920 & -3.535559 \\ \mathrm{O} & 1.450506 & 0.058313 & 4.840859 \\ \mathrm{H} & 0.450514 & 1.734099 & -4.301408 \\ \mathrm{H} & -0.454569 & 1.733041 & 5.606708 \\ \mathrm{H} & 1.243858 & 0.638056 & -5.470119 \\ \mathrm{H} & -1.245347 & 0.635145 & 6.775419 \\ \mathrm{H} & -1.263753 & 0.375607 & -5.527530 \\ \mathrm{H} & 1.262871 & 0.378561 & 6.832830 \\ \mathrm{H} & -0.475920 & -1.116309 & -4.932916 \\ \mathrm{H} & 0.478530 & -1.115193 & 6.238216\end{array}$

\section{TPP}

$\begin{array}{lrrr}\mathrm{P} & 1.610915 & 0.001441 & 0.001709 \\ \mathrm{~N} & 0.786103 & -1.358988 & -0.000367\end{array}$




\begin{tabular}{|c|c|c|c|}
\hline $\mathrm{P}$ & -0.804210 & -1.395813 & 0.001709 \\
\hline $\mathrm{N}$ & -1.569970 & -0.001291 & -0.000367 \\
\hline $\mathrm{P}$ & -0.806705 & 1. 394373 & 0.001709 \\
\hline $\mathrm{N}$ & 0.783867 & 1.360279 & -0.000367 \\
\hline 0 & 2. 708631 & 0.001714 & -1.211955 \\
\hline 0 & -1.352831 & -2.346601 & -1.211955 \\
\hline 0 & -1.355800 & 2.344887 & -1.211955 \\
\hline $\mathrm{C}$ & 3. 982971 & 0.001427 & -0.688293 \\
\hline $\mathrm{C}$ & -1.990250 & -3.450067 & -0.688293 \\
\hline $\mathrm{C}$ & -1.992721 & 3. 448641 & -0.688293 \\
\hline $\mathrm{C}$ & 3. 979849 & 0.001365 & 0.702600 \\
\hline $\mathrm{C}$ & -1.988742 & -3.447333 & 0.702600 \\
\hline $\mathrm{C}$ & -1.991107 & 3. 445967 & 0.702600 \\
\hline 0 & 2. 703272 & 0.001601 & 1. 220558 \\
\hline 0 & -1.350250 & -2.341903 & 1. 220558 \\
\hline 0 & -1.353022 & 2. 340302 & 1. 220558 \\
\hline $\mathrm{C}$ & 5. 156583 & 0.000343 & -1.411461 \\
\hline $\mathrm{C}$ & -2.577994 & -4.465904 & -1.411461 \\
\hline $\mathrm{C}$ & -2.578589 & 4. 465561 & -1.411461 \\
\hline $\mathrm{C}$ & 6. 351015 & -0.000831 & -0.683886 \\
\hline $\mathrm{C}$ & -3.176227 & -5.499725 & -0.683886 \\
\hline $\mathrm{C}$ & -3.174788 & 5.500556 & -0.683886 \\
\hline $\mathrm{C}$ & 6. 347925 & -0.000905 & 0.708613 \\
\hline $\mathrm{C}$ & -3.174746 & -5.497012 & 0.708613 \\
\hline $\mathrm{C}$ & -3.173179 & 5. 497917 & 0.708613 \\
\hline $\mathrm{C}$ & 5. 150297 & 0.000199 & 1. 430907 \\
\hline $\mathrm{C}$ & -2.574976 & -4.460387 & 1. 430907 \\
\hline $\mathrm{C}$ & -2.575321 & 4. 460188 & 1. 430907 \\
\hline $\mathrm{H}$ & 5. 140415 & 0.000208 & -2.495345 \\
\hline $\mathrm{H}$ & -2.570027 & -4.451834 & -2.495345 \\
\hline $\mathrm{H}$ & -2.570387 & 4. 451626 & -2.495345 \\
\hline $\mathrm{H}$ & 7. 295136 & -0.001738 & -1.218969 \\
\hline $\mathrm{H}$ & -3.649074 & -6.316904 & -1.218969 \\
\hline $\mathrm{H}$ & -3.646063 & 6.318643 & -1.218969 \\
\hline $\mathrm{H}$ & 7. 289672 & -0.001868 & 1. 247866 \\
\hline $\mathrm{H}$ & -3.646453 & -6.312107 & 1. 247866 \\
\hline $\mathrm{H}$ & -3.643219 & 6. 313975 & 1. 247866 \\
\hline $\mathrm{H}$ & 5. 129363 & -0.000038 & 2.514710 \\
\hline $\mathrm{H}$ & -2.564714 & -4.442139 & 2.514710 \\
\hline $\mathrm{H}$ & -2.564649 & 4. 442177 & 2. 514710 \\
\hline
\end{tabular}

\section{1a}

$\begin{array}{llll}\mathrm{C} & -0.519694 & 1.230661 & -0.684375 \\ 0 & -1.328156 & 0.165585 & -0.963389\end{array}$ 


\begin{tabular}{|c|c|c|c|}
\hline $\mathrm{C}$ & -0.805936 & -1.065399 & -0.684375 \\
\hline 0 & 0.520678 & -1.233010 & -0.963389 \\
\hline $\mathrm{C}$ & 1. 325631 & -0.165262 & -0.684375 \\
\hline 0 & 0.807479 & 1. 067425 & -0.963389 \\
\hline $\mathrm{C}$ & -1.012611 & 2. 399944 & -0.266828 \\
\hline $\mathrm{C}$ & -1.572107 & -2.076919 & -0.266828 \\
\hline $\mathrm{C}$ & 2. 584718 & -0.323025 & -0.266828 \\
\hline S & -2.732894 & 2. 663445 & 0.033771 \\
\hline S & -0.940164 & -3.698478 & 0.033771 \\
\hline S & 3. 673058 & 1. 035034 & 0.033771 \\
\hline $\mathrm{C}$ & -2.598123 & 4. 425273 & 0.103180 \\
\hline $\mathrm{C}$ & -2.533337 & -4.462677 & 0.103180 \\
\hline $\mathrm{C}$ & 5. 131460 & 0.037404 & 0.103180 \\
\hline $\mathrm{C}$ & -1.352083 & 4. 948729 & 0.110428 \\
\hline $\mathrm{C}$ & -3.609684 & -3.645303 & 0.110428 \\
\hline $\mathrm{C}$ & 4. 961767 & -1.303427 & 0.110428 \\
\hline S & 0.000000 & 3.821011 & 0.015635 \\
\hline S & -3.309092 & -1.910505 & 0.015635 \\
\hline S & 3. 309092 & -1.910505 & 0.015635 \\
\hline S & -4.088992 & 5. 337842 & 0.261270 \\
\hline S & -2.578211 & -6.210092 & 0.261270 \\
\hline S & 6. 667203 & 0.872250 & 0.261270 \\
\hline S & -0.913972 & 6.652738 & 0.180914 \\
\hline S & -5.304454 & -4.117892 & 0.180914 \\
\hline S & 6. 218427 & -2.534846 & 0.180914 \\
\hline $\mathrm{C}$ & -4.747709 & 5.207857 & -1.427929 \\
\hline $\mathrm{C}$ & -2.136282 & -6.715565 & -1.427929 \\
\hline $\mathrm{C}$ & 6. 883991 & 1. 507708 & -1.427929 \\
\hline $\mathrm{C}$ & -1.338046 & 7. 060873 & 1. 898893 \\
\hline $\mathrm{C}$ & -5.445872 & -4.689218 & 1. 898893 \\
\hline $\mathrm{C}$ & 6. 783918 & -2.371655 & 1.898893 \\
\hline $\mathrm{H}$ & -4.858884 & 4. 162897 & -1.722860 \\
\hline $\mathrm{H}$ & -1.175732 & -6.289366 & -1.722860 \\
\hline $\mathrm{H}$ & 6. 034616 & 2. 126469 & -1.722860 \\
\hline $\mathrm{H}$ & -5.731543 & 5.682178 & -1.409633 \\
\hline $\mathrm{H}$ & -2.055139 & -7.804751 & -1.409633 \\
\hline $\mathrm{H}$ & 7. 786682 & 2. 122573 & -1.409633 \\
\hline $\mathrm{H}$ & -4.102172 & 5. 735409 & -2.131756 \\
\hline $\mathrm{H}$ & -2.915924 & -6.420290 & -2.131756 \\
\hline $\mathrm{H}$ & 7. 018096 & 0.684881 & -2.131756 \\
\hline $\mathrm{H}$ & -0.991123 & 8.084605 & 2. 059582 \\
\hline $\mathrm{H}$ & -6.505912 & -4.900640 & 2. 059582 \\
\hline $\mathrm{H}$ & 7. 497034 & -3.183965 & 2. 059582 \\
\hline$H$ & -2.417367 & 7. 017817 & 2. 053339 \\
\hline
\end{tabular}




$\begin{array}{rrrr}\mathrm{H} & -4.868924 & -5.602409 & 2.053339 \\ \mathrm{H} & 7.286291 & -1.415407 & 2.053339 \\ \mathrm{H} & -0.821381 & 6.395760 & 2.592801 \\ \mathrm{H} & -5.128200 & -3.909217 & 2.592801 \\ \mathrm{H} & 5.949581 & -2.486543 & 2.592801\end{array}$

\section{1b}

$\begin{array}{lrrr}\text { C } & 1.332223 & -0.001475 & 0.589820 \\ \text { O } & 0.670900 & 1.159066 & 0.885020 \\ \mathrm{C} & -0.664834 & 1.154477 & 0.589820 \\ \mathrm{O} & -1.339230 & 0.001483 & 0.885020 \\ \mathrm{C} & -0.667389 & -1.153001 & 0.589820 \\ \mathrm{O} & 0.668331 & -1.160549 & 0.885020 \\ \mathrm{C} & 2.601286 & -0.002881 & 0.172967 \\ \mathrm{C} & -1.298148 & 2.254220 & 0.172967 \\ \mathrm{C} & -1.303138 & -2.251339 & 0.172967 \\ \mathrm{~S} & 3.510440 & 1.491175 & -0.097756 \\ \mathrm{~S} & -3.046616 & 2.294543 & -0.097756 \\ \mathrm{~S} & -0.463825 & -3.785718 & -0.097756 \\ \mathrm{C} & 4.963725 & 0.661793 & -0.612440 \\ \mathrm{C} & -3.054992 & 3.967815 & -0.612440 \\ \mathrm{C} & -1.908733 & -4.629608 & -0.612440 \\ \mathrm{C} & 4.962247 & -0.672786 & -0.612440 \\ \mathrm{C} & -1.898474 & 4.633825 & -0.612440 \\ \mathrm{C} & -3.063773 & -3.961039 & -0.612440 \\ \mathrm{~S} & 3.507129 & -1.498947 & -0.097756 \\ \mathrm{~S} & -0.455438 & 3.786736 & -0.097756 \\ \mathrm{~S} & -3.051691 & -2.287789 & -0.097756 \\ \mathrm{H} & 5.805285 & 1.277363 & -0.906544 \\ \mathrm{H} & -4.008872 & 4.388843 & -0.906544 \\ \mathrm{H} & -1.796414 & -5.666206 & -0.906544 \\ \mathrm{H} & 5.802442 & -1.290219 & -0.906544 \\ \mathrm{H} & -1.783859 & 5.670171 & -0.906544 \\ \mathrm{H} & -4.018583 & -4.379953 & -0.906544\end{array}$

\section{1c}

$\begin{array}{rrrr}\mathrm{C} & 1.302775 & 0.000228 & 1.216033 \\ 0 & 0.666852 & 1.155488 & 1.604972 \\ \mathrm{C} & -0.651585 & 1.128122 & 1.216033 \\ 0 & -1.334108 & -0.000233 & 1.604972 \\ \mathrm{C} & -0.651190 & -1.128350 & 1.216033 \\ 0 & 0.667256 & -1.155255 & 1.604972 \\ \mathrm{C} & 2.419297 & 0.000423 & 0.495176 \\ \mathrm{C} & -1.210015 & 2.094961 & 0.495176\end{array}$




$\begin{array}{rrrr}\mathrm{C} & -1.209282 & -2.095384 & 0.495176 \\ \mathrm{Se} & 3.249004 & -1.602251 & -0.064422 \\ \mathrm{Se} & -0.236913 & 3.614846 & -0.064422 \\ \mathrm{Se} & -3.012092 & -2.012595 & -0.064422 \\ \mathrm{C} & 4.621670 & -0.665967 & -0.945663 \\ \mathrm{C} & -1.734091 & 4.335468 & -0.945663 \\ \mathrm{C} & -2.887580 & -3.669500 & -0.945663 \\ \mathrm{C} & 4.621437 & 0.667584 & -0.945663 \\ \mathrm{C} & -2.888864 & 3.668490 & -0.945663 \\ \mathrm{C} & -1.732574 & -4.336074 & -0.945663 \\ \mathrm{Se} & 3.248444 & 1.603387 & -0.064422 \\ \mathrm{Se} & -3.012796 & 2.011541 & -0.064422 \\ \mathrm{Se} & -0.235648 & -3.614928 & -0.064422 \\ \mathrm{H} & 5.382321 & -1.263259 & -1.435608 \\ \mathrm{H} & -1.597146 & 5.292857 & -1.435608 \\ \mathrm{H} & -3.785175 & -4.029598 & -1.435608 \\ \mathrm{H} & 5.381879 & 1.265142 & -1.435608 \\ \mathrm{H} & -3.786585 & 4.028273 & -1.435608 \\ \mathrm{H} & -1.595294 & -5.293415 & -1.435608\end{array}$

\section{1d}

C $\quad 0.182990$

1. 325341

$-1.251514$

$0 \quad-1.056641$

0.821353

$-1.521511$

C $\quad-1.239274$

$-0.504196$

$-1.251514$

$0 \quad-0.182992$

$-1.325755$

$-1.521511$

C $\quad 1.056284$

$-0.821144$

$-1.251514$

o 1.239633

0.504402

$-1.521511$

C $\quad 0.359239$

2. 606502

$-0.914530$

C $\quad-2.436917$

$-0.992141$

$-0.914530$

C $\quad 2.077678$

$-1.614361$

$-0.914530$

S $\quad 1.958327$

3. 328332

$-0.722300$

S $\quad-3.861584$

0.031795

$-0.722300$

1. 903256

$-3.360127$

$-0.722300$

C $\quad 1.336513$

4. 687577

0. 227141

C $\quad-4.727818$

$-1.186334$

0. 227141

3. 391304

$-3.501243$

0. 227141

C $\quad 0.002432$

4. 872006

0. 227742

C $\quad-4.220497$

$-2.433897$

0. 227742

C $\quad 4.218065$

$-2.438109$

0. 227742

S $\quad-0.976775$

3. 739944

$-0.714439$

S $\quad-2.750499$

$-2.715884$

$-0.714439$

S $\quad 3.727274$

$-1.024060$

$-0.714439$

S $\quad-0.804817$

6. 251700

0.942775

S $\quad-5.011722$

$-3.822841$

0.942775 


$\begin{array}{rrrr}\text { S } & 5.816539 & -2.428858 & 0.942775 \\ \mathrm{C} & 0.469049 & 7.478201 & 0.509559 \\ \mathrm{C} & -6.710837 & -3.332893 & 0.509559 \\ \mathrm{C} & 6.241788 & -4.145309 & 0.509559 \\ \mathrm{C} & 1.757229 & 7.270157 & 1.278687 \\ \mathrm{C} & -7.174756 & -2.113274 & 1.278687 \\ \mathrm{C} & 5.417526 & -5.156884 & 1.278687 \\ \mathrm{~S} & 2.574046 & 5.647422 & 1.012374 \\ \mathrm{~S} & -6.177834 & -0.594521 & 1.012374 \\ \mathrm{~S} & 3.603787 & -5.052901 & 1.012374 \\ \mathrm{H} & 0.628245 & 7.444069 & -0.571320 \\ \mathrm{H} & -6.760875 & -3.177958 & -0.571320 \\ \mathrm{H} & 6.132630 & -4.266111 & -0.571320 \\ \mathrm{H} & 0.051074 & 8.455404 & 0.769998 \\ \mathrm{H} & -7.348132 & -4.183471 & 0.769998 \\ \mathrm{H} & 7.297058 & -4.271933 & 0.769998 \\ \mathrm{H} & 1.572335 & 7.370233 & 2.350117 \\ \mathrm{H} & -7.168976 & -2.323434 & 2.350117 \\ \mathrm{H} & 5.596641 & -5.046799 & 2.350117 \\ \mathrm{H} & 2.495494 & 8.021658 & 0.978856 \\ \mathrm{H} & -8.194707 & -1.849667 & 0.978856 \\ \mathrm{H} & 5.699212 & -6.171991 & 0.978856\end{array}$

$1 e$

$\begin{array}{rrrr}\text { C } & 1.290957 & 0.002667 & -2.478284 \\ 0 & 0.662942 & 1.154198 & -2.893820 \\ \mathrm{C} & -0.647788 & 1.116668 & -2.478284 \\ 0 & -1.331036 & -0.002975 & -2.893820 \\ \mathrm{C} & -0.643169 & -1.119335 & -2.478284 \\ \mathrm{O} & 0.668094 & -1.151224 & -2.893820 \\ \mathrm{C} & 2.359055 & 0.006432 & -1.689509 \\ \mathrm{C} & -1.185098 & 2.039786 & -1.689509 \\ \mathrm{C} & -1.173957 & -2.046218 & -1.689509 \\ \mathrm{Se} & 3.180986 & -1.578094 & -1.071437 \\ \mathrm{Se} & -0.223824 & 3.543862 & -1.071437 \\ \mathrm{Se} & -2.957162 & -1.965768 & -1.071437 \\ \mathrm{C} & 3.809987 & -0.667368 & 0.474057 \\ \mathrm{C} & -1.327035 & 3.633230 & 0.474057 \\ \mathrm{C} & -2.482951 & -2.965861 & 0.474057 \\ \mathrm{C} & 3.803625 & 0.678314 & 0.472205 \\ \mathrm{C} & -2.489250 & 2.954878 & 0.472205 \\ \mathrm{C} & -1.314375 & -3.633193 & 0.472205 \\ \mathrm{Se} & 3.174797 & 1.591167 & -1.067558 \\ \mathrm{Se} & -2.965390 & 1.953871 & -1.067558\end{array}$




$\begin{array}{rrrr}\text { Se } & -0.209407 & -3.545039 & -1.067558 \\ \mathrm{~S} & 4.512341 & 1.643678 & 1.745917 \\ \mathrm{~S} & -3.679637 & 3.085962 & 1.745917 \\ \mathrm{~S} & -0.832703 & -4.729641 & 1.745917 \\ \mathrm{C} & 5.970457 & 0.582208 & 2.011878 \\ \mathrm{C} & -3.489435 & 4.879464 & 2.011878 \\ \mathrm{C} & -2.481022 & -5.461671 & 2.011878 \\ \mathrm{C} & 5.619327 & -0.746187 & 2.648271 \\ \mathrm{C} & -2.163446 & 5.239573 & 2.648271 \\ \mathrm{C} & -3.455880 & -4.493386 & 2.648271 \\ \mathrm{~S} & 4.347738 & -1.729633 & 1.753718 \\ \mathrm{~S} & -0.675962 & 4.630068 & 1.753718 \\ \mathrm{~S} & -3.671775 & -2.900434 & 1.753718 \\ \mathrm{H} & 6.469060 & 0.450112 & 1.048095 \\ \mathrm{H} & -3.624339 & 5.377314 & 1.048095 \\ \mathrm{H} & -2.844721 & -5.827427 & 1.048095 \\ \mathrm{H} & 6.639840 & 1.134820 & 2.678299 \\ \mathrm{H} & -4.302703 & 5.182860 & 2.678299 \\ \mathrm{H} & -2.337137 & -6.317680 & 2.678299 \\ \mathrm{H} & 5.237311 & -0.589013 & 3.659313 \\ \mathrm{H} & -2.108555 & 4.830151 & 3.659313 \\ \mathrm{H} & -3.128756 & -4.241137 & 3.659313 \\ \mathrm{H} & 6.513309 & -1.376696 & 2.703986 \\ \mathrm{H} & -2.064401 & 6.329039 & 2.703986 \\ \mathrm{H} & -4.448908 & -4.952344 & 2.703986\end{array}$

1f

$\begin{array}{lrrr}\text { C } & 1.332096 & -0.003112 & -0.999004 \\ \text { O } & 0.672083 & 1.158043 & -1.293140 \\ \mathrm{C} & -0.663353 & 1.155185 & -0.999004 \\ 0 & -1.338936 & 0.003019 & -1.293140 \\ \mathrm{C} & -0.668744 & -1.152073 & -0.999004 \\ 0 & 0.666853 & -1.161063 & -1.293140 \\ \mathrm{C} & 2.603682 & -0.005976 & -0.589822 \\ \mathrm{C} & -1.296666 & 2.257843 & -0.589822 \\ \mathrm{C} & -1.307016 & -2.251867 & -0.589822 \\ \mathrm{~S} & 3.489442 & -1.520023 & -0.343421 \\ \mathrm{~S} & -0.428343 & 3.781957 & -0.343421 \\ \mathrm{~S} & -3.061100 & -2.261934 & -0.343421 \\ \mathrm{C} & 4.924890 & -0.681954 & 0.236081 \\ \mathrm{C} & -1.871855 & 4.606057 & 0.236081 \\ \mathrm{C} & -3.053035 & -3.924102 & 0.236081 \\ \mathrm{C} & 4.926733 & 0.657506 & 0.240021 \\ \mathrm{C} & -3.032784 & 3.937923 & 0.240021\end{array}$




$\begin{array}{rrrr}\mathrm{C} & -1.893949 & -4.595429 & 0.240021 \\ \mathrm{~S} & 3.495611 & 1.503424 & -0.337852 \\ \mathrm{~S} & -3.049809 & 2.275576 & -0.337852 \\ \mathrm{~S} & -0.445802 & -3.779000 & -0.337852 \\ \mathrm{O} & 5.976519 & 1.427243 & 0.632481 \\ \mathrm{O} & -4.224288 & 4.462196 & 0.632481 \\ \mathrm{O} & -1.752231 & -5.889438 & 0.632481 \\ \mathrm{C} & 7.160661 & 0.640589 & 0.697866 \\ \mathrm{C} & -4.135097 & 5.881020 & 0.697866 \\ \mathrm{C} & -3.025564 & -6.521609 & 0.697866 \\ \mathrm{C} & 6.884399 & -0.676382 & 1.406688 \\ \mathrm{C} & -2.856436 & 6.300255 & 1.406688 \\ \mathrm{C} & -4.027963 & -5.623874 & 1.406688 \\ \mathrm{O} & 5.956262 & -1.457951 & 0.663226 \\ \mathrm{O} & -1.715508 & 5.887250 & 0.663226 \\ \mathrm{O} & -4.240754 & -4.429299 & 0.663226 \\ \mathrm{H} & 7.526675 & 0.444824 & -0.318255 \\ \mathrm{H} & -4.148567 & 6.295880 & -0.318255 \\ \mathrm{H} & -3.378108 & -6.740704 & -0.318255 \\ \mathrm{H} & 7.897680 & 1.233278 & 1.243792 \\ \mathrm{H} & -5.016890 & 6.222953 & 1.243792 \\ \mathrm{H} & -2.880790 & -7.456231 & 1.243792 \\ \mathrm{H} & 6.477671 & -0.479293 & 2.406968 \\ \mathrm{H} & -2.823756 & 5.849474 & 2.406968 \\ \mathrm{H} & -3.653915 & -5.370181 & 2.406968 \\ \mathrm{H} & 7.794108 & -1.274207 & 1.494129 \\ \mathrm{H} & -2.793558 & 7.386999 & 1.494129 \\ \mathrm{H} & -5.000550 & -6.112792 & 1.494129\end{array}$

2b

$\begin{array}{lrrr}\text { C } & 1.566976 & -0.001868 & 1.718019 \\ \mathrm{~S} & 0.880162 & 1.520464 & 2.308666 \\ \mathrm{C} & -0.781870 & 1.357975 & 1.718019 \\ \mathrm{~S} & -1.756841 & 0.002010 & 2.308666 \\ \mathrm{C} & -0.785106 & -1.356107 & 1.718019 \\ \mathrm{~S} & 0.876680 & -1.522474 & 2.308666 \\ \mathrm{C} & 2.668633 & -0.003047 & 0.930548 \\ \mathrm{C} & -1.331677 & 2.312628 & 0.930548 \\ \mathrm{C} & -1.336956 & -2.309580 & 0.930548 \\ \mathrm{~S} & 3.462125 & 1.476697 & 0.379604 \\ \mathrm{~S} & -3.009919 & 2.259940 & 0.379604 \\ \mathrm{~S} & -0.452206 & -3.736637 & 0.379604 \\ \mathrm{C} & 4.690349 & 0.662449 & -0.558355 \\ \mathrm{C} & -2.918872 & 3.730737 & -0.558355\end{array}$




$\begin{array}{rrrr}\text { C } & -1.771477 & -4.393186 & -0.558355 \\ \mathrm{C} & 4.689475 & -0.672948 & -0.557522 \\ \mathrm{C} & -1.761948 & 4.397679 & -0.557522 \\ \mathrm{C} & -2.927527 & -3.724731 & -0.557522 \\ \mathrm{~S} & 3.460156 & -1.484510 & 0.381274 \\ \mathrm{~S} & -0.444454 & 3.738838 & 0.381274 \\ \mathrm{~S} & -3.015702 & -2.254327 & 0.381274 \\ \mathrm{H} & 5.397700 & 1.282358 & -1.096180 \\ \mathrm{H} & -3.809405 & 4.033366 & -1.096180 \\ \mathrm{H} & -1.588295 & -5.315724 & -1.096180 \\ \mathrm{H} & 5.396028 & -1.294435 & -1.094599 \\ \mathrm{H} & -1.577001 & 5.320315 & -1.094599 \\ \mathrm{H} & -3.819027 & -4.025880 & -1.094599\end{array}$

3b

$\begin{array}{llll}\text { C } & 1.405525 & 0.000152 & -0.430437\end{array}$

$\begin{array}{llll}\mathrm{N} & 0.674277 & 1.168173 & -0.485480\end{array}$

$\begin{array}{llll}\text { C } & -0.702894 & 1.217145 & -0.430437\end{array}$

$\begin{array}{llll}\mathrm{N} & -1.348806 & -0.000146 & -0.485480\end{array}$

$\begin{array}{llll}\text { C } & -0.702631 & -1.217296 & -0.430437\end{array}$

$\begin{array}{llll}\mathrm{N} & 0.674529 & -1.168028 & -0.485480\end{array}$

$\begin{array}{llll}\text { C } & 2.762939 & 0.000298 & -0.383763\end{array}$

$\begin{array}{llll}\text { C } & -1.381728 & 2.392626 & -0.383763\end{array}$

$\begin{array}{llll}\text { C } & -1.381211 & -2.392924 & -0.383763\end{array}$

$\begin{array}{llll}\text { S } & 3.725744 & 1.467865 & -0.183443\end{array}$

$\begin{array}{llll}\mathrm{S} & -3.134080 & 2.492656 & -0.183443\end{array}$

$\begin{array}{llll}\text { S } & -0.591664 & -3.960522 & -0.183443\end{array}$

$\begin{array}{llll}\text { C } & 4.856146 & 0.668505 & 0.912839\end{array}$

$\begin{array}{lrrr}\text { C } & -3.007016 & 3.871293 & 0.912839\end{array}$

$\begin{array}{llll}\text { C } & -1.849131 & -4.539799 & 0.912839\end{array}$

$\begin{array}{llll}\text { C } & 4.856291 & -0.667457 & 0.912839\end{array}$

$\begin{array}{llll}\text { C } & -1.850111 & 4.539399 & 0.912839\end{array}$

$\begin{array}{llll}\text { C } & -3.006180 & -3.871943 & 0.912839\end{array}$

$\begin{array}{llll}\text { S } & 3.726061 & -1.467060 & -0.183443\end{array}$

$\begin{array}{lrrr}\text { S } & -0.592519 & 3.960394 & -0.183443\end{array}$

$\begin{array}{llll}\mathrm{S} & -3.133542 & -2.493333 & -0.183443\end{array}$

$\begin{array}{llll}\mathrm{H} & 5.542347 & 1.286123 & 1.479863\end{array}$

$\mathrm{H} \quad-3.884989 \quad 4.156752 \quad 1.479863$

$\begin{array}{llll}\mathrm{H} & -1.657358 & -5.442875 & 1.479863\end{array}$

$\begin{array}{lrrr}\mathrm{H} & 5.542625 & -1.284926 & 1.479863\end{array}$

$\begin{array}{lrrr}\mathrm{H} & -1.658534 & 5.442517 & 1.479863\end{array}$

H $\quad-3.884091 \quad-4.157591 \quad 1.479863$

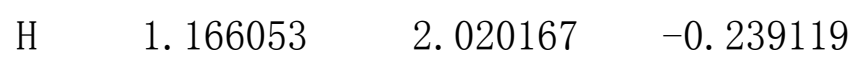

$\begin{array}{llll}\mathrm{H} & -2.332542 & -0.000252 & -0.239119\end{array}$ 
H $\quad 1.166489 \quad-2.019915 \quad-0.239119$

4b

$\begin{array}{lrrr}\mathrm{P} & 1.611752 & -0.000393 & 0.910800 \\ \mathrm{~N} & 0.809084 & 1.400587 & 0.742248 \\ \mathrm{P} & -0.805536 & 1.396015 & 0.910800 \\ \mathrm{~N} & -1.617486 & 0.000394 & 0.742248 \\ \mathrm{P} & -0.806216 & -1.395622 & 0.910800 \\ \mathrm{~N} & 0.808401 & -1.400981 & 0.742248 \\ \mathrm{C} & 3.129544 & -0.000763 & 0.110944 \\ \mathrm{C} & -1.564111 & 2.710646 & 0.110944 \\ \mathrm{C} & -1.565433 & -2.709883 & 0.110944 \\ \mathrm{~S} & 4.052772 & -1.464658 & -0.097790 \\ \mathrm{~S} & -0.757955 & 4.242133 & -0.097790 \\ \mathrm{~S} & -3.294817 & -2.777475 & -0.097790 \\ \mathrm{C} & 5.610735 & -0.670343 & 0.006296 \\ \mathrm{C} & -2.224834 & 5.194210 & 0.006296 \\ \mathrm{C} & -3.385901 & -4.523867 & 0.006296 \\ \mathrm{C} & 5.611061 & 0.667606 & 0.006296 \\ \mathrm{C} & -3.383694 & 4.525518 & 0.006296 \\ \mathrm{C} & -2.227367 & -5.193124 & 0.006296 \\ \mathrm{~S} & 4.053486 & 1.462681 & -0.097790 \\ \mathrm{~S} & -3.293462 & 2.779081 & -0.097790 \\ \mathrm{~S} & -0.760024 & -4.241763 & -0.097790 \\ \mathrm{H} & 6.498314 & -1.291641 & 0.027565 \\ \mathrm{H} & -2.130563 & 6.273526 & 0.027565 \\ \mathrm{H} & -4.367751 & -4.981885 & 0.027565 \\ \mathrm{H} & 6.498944 & 1.288472 & 0.027565 \\ \mathrm{H} & -4.365321 & 4.984014 & 0.027565 \\ \mathrm{H} & -2.133622 & -6.272486 & 0.027565\end{array}$

\section{4h1}

$\mathrm{P}$

0.807866

1. 392687

$-0.000188$

$\mathrm{N} \quad-0.782377$

1. 360951

$-0.000224$

P $\quad-1.610036$

0. 003289

$-0.000188$

$\mathrm{N} \quad-0.787429$

$-1.358034$

$-0.000224$

P $\quad 0.802170$

$-1.395976$

$-0.000188$

$\mathrm{N} \quad 1.569807$

$-0.002917$

$-0.000224$

$0 \quad 1.357816$

2. 340569

1. 216252

$0 \quad-2.705900$

0. 005619

1. 216252

$0 \quad 1.348084$

$-2.346188$

1. 216252

C $\quad 1.989723$

3. 446301

0.695572

C $\quad-3.979446$

0.000000

0.695572

C $\quad 1.989723$

$-3.446301$

0. 695572 


\begin{tabular}{|c|c|c|c|}
\hline $\mathrm{C}$ & 1. 989746 & 3. 446340 & -0.695786 \\
\hline $\mathrm{C}$ & -3.979491 & 0.000000 & -0.695786 \\
\hline $\mathrm{C}$ & 1. 989745 & -3.446340 & -0.695786 \\
\hline 0 & 1. 357857 & 2. 340638 & -1.216550 \\
\hline 0 & -2.705980 & 0.005620 & -1.216550 \\
\hline 0 & 1. 348123 & -2.346257 & -1.216550 \\
\hline C & 2.560695 & 4. 464819 & 1. 427600 \\
\hline $\mathrm{C}$ & -5.146994 & -0.014783 & 1. 427600 \\
\hline C & 2. 586299 & -4.450036 & 1. 427600 \\
\hline C & 3. 154416 & 5.500819 & 0.700685 \\
\hline C & -6.341057 & -0.018605 & 0.700685 \\
\hline $\mathrm{C}$ & 3. 186641 & -5.482213 & 0.700685 \\
\hline $\mathrm{C}$ & 3. 154437 & 5.500853 & -0.700740 \\
\hline C & -6.341097 & -0.018604 & -0.700740 \\
\hline $\mathrm{C}$ & 3. 186660 & -5.482249 & -0.700740 \\
\hline $\mathrm{C}$ & 2. 560741 & 4. 464899 & -1.427737 \\
\hline C & -5.147086 & -0.014782 & -1.427737 \\
\hline $\mathrm{C}$ & 2.586345 & -4.450116 & -1.427737 \\
\hline S & 3. 990361 & 6.843012 & 1. 479098 \\
\hline S & -7.921403 & 0.034248 & 1. 479098 \\
\hline$S$ & 3. 931042 & -6.877260 & 1. 479098 \\
\hline $\mathrm{C}$ & 4. 132095 & 7. 794859 & 0.000063 \\
\hline $\mathrm{C}$ & -8.816594 & -0.318930 & 0.000063 \\
\hline $\mathrm{C}$ & 4. 684499 & -7.475929 & 0.000063 \\
\hline S & 3. 990437 & 6.843074 & -1.479051 \\
\hline $\mathrm{S}$ & -7.921494 & 0.034283 & -1.479051 \\
\hline S & 3.931058 & -6.877357 & -1.479051 \\
\hline $\mathrm{C}$ & 4. 360328 & 9. 124203 & 0.000108 \\
\hline $\mathrm{C}$ & -10.081956 & -0.785947 & 0.000108 \\
\hline $\mathrm{C}$ & 5. 721628 & -8.338257 & 0.000108 \\
\hline S & 4. 530102 & 10. 072895 & -1.482372 \\
\hline S & -10.988434 & -1.113264 & -1.482372 \\
\hline S & 6. 458332 & -8.959631 & -1.482372 \\
\hline $\mathrm{C}$ & 4. 475612 & 11. 619689 & -0.667357 \\
\hline $\mathrm{C}$ & -12.300752 & -1.933851 & -0.667357 \\
\hline $\mathrm{C}$ & 7. 825140 & -9.685838 & -0.667357 \\
\hline $\mathrm{C}$ & 4. 475568 & 11.619633 & 0.667762 \\
\hline $\mathrm{C}$ & -12.300681 & -1.933861 & 0.667762 \\
\hline $\mathrm{C}$ & 7. 825114 & -9.685772 & 0.667762 \\
\hline S & 4.530000 & 10.072774 & 1. 482662 \\
\hline S & -10.988278 & -1.113292 & 1. 482662 \\
\hline $\mathrm{S}$ & 6.458278 & -8.959482 & 1. 482662 \\
\hline 11 & 4. 470878 & 12.508305 & 1. 286454 \\
\hline$H$ & -13.067948 & -2.382258 & 1. 286454 \\
\hline
\end{tabular}




$\begin{array}{rrrr}\mathrm{H} & 8.597071 & -10.126046 & 1.286454 \\ \mathrm{H} & 4.470963 & 12.508414 & -1.285972 \\ \mathrm{H} & -13.068086 & -2.382239 & -1.285972 \\ \mathrm{H} & 8.597123 & -10.126175 & -1.285972 \\ \mathrm{H} & 2.541834 & 4.455275 & 2.510837 \\ \mathrm{H} & -5.129299 & -0.026344 & 2.510837 \\ \mathrm{H} & 2.587464 & -4.428931 & 2.510837 \\ \mathrm{H} & 2.541918 & 4.455419 & -2.510975 \\ \mathrm{H} & -5.129465 & -0.026344 & -2.510975 \\ \mathrm{H} & 2.587547 & -4.429075 & -2.510975\end{array}$

\section{4h2}

$\begin{array}{lrrr}\mathrm{P} & 0.370342 & 1.582460 & -0.000195 \\ \mathrm{~N} & -1.154080 & 1.089463 & -0.000232 \\ \mathrm{P} & -1.555622 & -0.470504 & -0.000195 \\ \mathrm{~N} & -0.366462 & -1.544194 & -0.000232 \\ \mathrm{P} & 1.185280 & -1.111956 & -0.000195 \\ \mathrm{~N} & 1.520543 & 0.454732 & -0.000232 \\ \mathrm{~S} & 0.669066 & 2.936841 & 1.580939 \\ \mathrm{~S} & -2.877912 & -0.888993 & 1.580939 \\ \mathrm{~S} & 2.208846 & -2.047848 & 1.580939 \\ \mathrm{C} & 1.832880 & 3.936499 & 0.703025 \\ \mathrm{C} & -4.325548 & -0.380929 & 0.703025 \\ \mathrm{C} & 2.492669 & -3.555570 & 0.703025 \\ \mathrm{C} & 1.832927 & 3.936518 & -0.703242 \\ \mathrm{C} & -4.325588 & -0.380898 & -0.703242 \\ \mathrm{C} & 2.492661 & -3.555620 & -0.703242 \\ \mathrm{~S} & 0.669203 & 2.936888 & -1.581288 \\ \mathrm{~S} & -2.878021 & -0.888897 & -1.581288 \\ \mathrm{~S} & 2.208818 & -2.047991 & -1.581288 \\ \mathrm{C} & 2.735012 & 4.737289 & 1.402161 \\ \mathrm{C} & -5.470118 & -0.000055 & 1.402161 \\ \mathrm{C} & 2.735107 & -4.737234 & 1.402161 \\ \mathrm{C} & 3.604138 & 5.562880 & 0.700904 \\ \mathrm{C} & -6.619664 & 0.339835 & 0.700904 \\ \mathrm{C} & 3.015526 & -5.902715 & 0.700904 \\ \mathrm{C} & 3.604185 & 5.562896 & -0.700919 \\ \mathrm{C} & -6.619701 & 0.339868 & -0.700919 \\ \mathrm{C} & 3.015517 & -5.902763 & -0.700919 \\ \mathrm{C} & 2.735107 & 4.737343 & -1.402278 \\ \mathrm{C} & -5.470213 & 0.000000 & -1.402278 \\ \mathrm{C} & 2.735106 & -4.737343 & -1.402278 \\ \mathrm{~S} & 4.722961 & 6.674736 & 1.480917 \\ \mathrm{~S} & -8.141971 & 0.752836 & 1.480917 \\ & & & \\ \mathrm{H} & -1.59 & \end{array}$




$\begin{array}{rrrr}\mathrm{S} & 3.419010 & -7.427572 & 1.480917 \\ \mathrm{C} & 5.612967 & 7.038289 & 0.000056 \\ \mathrm{C} & -8.901820 & 1.341827 & 0.000056 \\ \mathrm{C} & 3.288854 & -8.380116 & 0.000056 \\ \mathrm{~S} & 4.723011 & 6.674832 & -1.480849 \\ \mathrm{~S} & -8.142079 & 0.752832 & -1.480849 \\ \mathrm{~S} & 3.419068 & -7.427663 & -1.480849 \\ \mathrm{C} & 6.850673 & 7.574003 & 0.000143 \\ \mathrm{C} & -9.984616 & 2.145855 & 0.000143 \\ \mathrm{C} & 3.133943 & -9.719858 & 0.000143 \\ \mathrm{~S} & 7.732971 & 7.961698 & 1.482647 \\ \mathrm{~S} & -10.761518 & 2.716100 & 1.482647 \\ \mathrm{~S} & 3.028547 & -10.677798 & 1.482647 \\ \mathrm{C} & 9.248551 & 8.275745 & 0.667855 \\ \mathrm{C} & -11.791280 & 3.871608 & 0.667855 \\ \mathrm{C} & 2.542729 & -12.147352 & 0.667855 \\ \mathrm{C} & 9.248599 & 8.275837 & -0.667358 \\ \mathrm{C} & -11.791385 & 3.871603 & -0.667358 \\ \mathrm{C} & 2.542786 & -12.147440 & -0.667358 \\ \mathrm{~S} & 7.732996 & 7.962018 & -1.482239 \\ \mathrm{~S} & -10.761808 & 2.715961 & -1.482239 \\ \mathrm{~S} & 3.028812 & -10.677980 & -1.482239 \\ \mathrm{H} & 10.113273 & 8.481550 & 1.286843 \\ \mathrm{H} & -12.401874 & 4.517576 & 1.286843 \\ \mathrm{H} & 2.288601 & -12.999126 & 1.286843 \\ \mathrm{H} & 10.113351 & 8.481710 & -1.286276 \\ \mathrm{H} & -12.402052 & 4.517564 & -1.286276 \\ \mathrm{H} & 2.288701 & -12.999274 & -1.286276 \\ \mathrm{H} & 2.757006 & 4.710604 & -2.486746 \\ \mathrm{H} & -5.458006 & 0.032335 & -2.486746 \\ \mathrm{H} & 2.701000 & -4.742940 & -2.486746 \\ \mathrm{H} & 2.756837 & 4.710478 & 2.486629 \\ \mathrm{H} & -5.457812 & 0.032252 & 2.486629 \\ \mathrm{H} & 2.700975 & -4.742730 & 2.486629\end{array}$

\section{4h3}

$\begin{array}{lrrr}\text { P } & -0.064782 & 1.641407 & 0.041725 \\ \text { N } & 1.327348 & 0.837590 & 0.041103 \\ \text { P } & 1.453891 & -0.764600 & 0.041725 \\ \text { N } & 0.061700 & -1.568312 & 0.041103 \\ \text { P } & -1.389109 & -0.876807 & 0.041725 \\ \text { N } & -1.389048 & 0.730722 & 0.041103 \\ \text { N } & -0.205562 & 2.821206 & -1.158219 \\ \text { N } & 2.546017 & -1.232581 & -1.158219\end{array}$




\begin{tabular}{|c|c|c|c|}
\hline $\mathrm{N}$ & -2.340455 & -1.588625 & -1.158219 \\
\hline $\mathrm{C}$ & -0.225483 & 4. 122954 & -0.655338 \\
\hline $\mathrm{C}$ & 3. 683325 & -1.866203 & -0.655338 \\
\hline$C$ & -3.457841 & -2.256751 & -0.655338 \\
\hline $\mathrm{C}$ & -0.077063 & 4. 126339 & 0.743854 \\
\hline $\mathrm{C}$ & 3. 612045 & -1.996431 & 0.74 \\
\hline $\mathrm{C}$ & -3.534983 & -2.129907 & 0.7 \\
\hline $\mathrm{N}$ & -0.014722 & 2. 825321 & 1.24 \\
\hline $\mathrm{N}$ & 2. 454161 & -1.399911 & 1.24 \\
\hline $\mathrm{N}$ & -2.439439 & -1.425411 & 1.24 \\
\hline C & -0.335923 & 5. 307380 & -1.3 \\
\hline $\mathrm{C}$ & 4. 764288 & -2.362772 & -1.36 \\
\hline $\mathrm{C}$ & -4.428365 & -2.944609 & -1.36 \\
\hline $\mathrm{C}$ & -0.293773 & 6.507073 & -0.64 \\
\hline C & 5. 782178 & -2.999121 & -0.6 \\
\hline $\mathrm{C}$ & -5.488404 & -3.507952 & -0.64 \\
\hline $\mathrm{C}$ & -0.126359 & 6.510754 & \\
\hline $\mathrm{C}$ & 5.701657 & -3.145947 & 0.73 \\
\hline $\mathrm{C}$ & -5.575299 & -3.364807 & 0.73 \\
\hline $\mathrm{C}$ & -0.015114 & 5.3 & \\
\hline $\mathrm{C}$ & 4. 609899 & -2.644075 & 1. 45118 \\
\hline $\mathrm{C}$ & -4.594786 & -2.670253 & 1. 45118 \\
\hline S & -0.487626 & 8. 083884 & -1.41 \\
\hline S & 7. 244662 & -3.619646 & -1.41572 \\
\hline S & -6.757036 & -4.464238 & $-1.415^{\prime}$ \\
\hline $\mathrm{C}$ & 0.000000 & 8. 996928 & 0.012 \\
\hline $\mathrm{C}$ & 7. 791568 & -4.498464 & 0.01293 \\
\hline $\mathrm{C}$ & -7.791568 & -4.498464 & 0.01293 \\
\hline S & -0.131464 & 8. 091536 & 1.5215 \\
\hline S & 7. 073208 & -3.931917 & 1.52151 \\
\hline S & -6.941744 & -4.159619 & 1.52151 \\
\hline $\mathrm{C}$ & 0.402765 & 10. 283074 & -0.0382 \\
\hline $\mathrm{C}$ & 8. 704021 & -5.490342 & -0.0382 \\
\hline $\mathrm{C}$ & -9.106786 & -4.792732 & -0.03829 \\
\hline S & 0.506793 & 11. 203124 & -1.54597 \\
\hline S & 9.448793 & -6.040458 & -1.5459 \\
\hline S & -9.955586 & -5.162666 & $-1.5459^{\prime}$ \\
\hline $\mathrm{C}$ & 1. 375578 & 12.543468 & -0.8319 \\
\hline $\mathrm{C}$ & 10. 175173 & -7.463019 & -0.8319 \\
\hline $\mathrm{C}$ & -11.550751 & -5.080449 & -0.8319 \\
\hline $\mathrm{C}$ & 1. 536426 & 12.545260 & 0.4937 \\
\hline $\mathrm{C}$ & 10. 096301 & -7.603214 & 0.4937 \\
\hline $\mathrm{C}$ & -11.632727 & -4.942046 & 0.4937 \\
\hline S & 0.863705 & 11. 207214 & 1.3983 \\
\hline
\end{tabular}




$\begin{array}{rrrr}\text { S } & 9.273880 & -6.351598 & 1.398318 \\ \mathrm{~S} & -10.137585 & -4.855616 & 1.398318 \\ \mathrm{H} & -0.661717 & 2.605202 & -2.030502 \\ \mathrm{H} & 2.587029 & -0.729537 & -2.030502 \\ \mathrm{H} & -1.925313 & -1.875664 & -2.030502 \\ \mathrm{H} & -0.433225 & 5.305266 & -2.444022 \\ \mathrm{H} & 4.811108 & -2.277449 & -2.444022 \\ \mathrm{H} & -4.377883 & -3.027817 & -2.444022 \\ \mathrm{H} & 1.720122 & 13.325520 & -1.497564 \\ \mathrm{H} & 10.680178 & -8.152429 & -1.497564 \\ \mathrm{H} & -12.400300 & -5.173091 & -1.497564 \\ \mathrm{H} & 2.029882 & 13.328962 & 1.055590 \\ \mathrm{H} & 10.528278 & -8.422410 & 1.055590 \\ \mathrm{H} & -12.558160 & -4.906551 & 1.055590 \\ \mathrm{H} & 0.108957 & 5.316930 & 2.529349 \\ \mathrm{H} & 4.550118 & -2.752824 & 2.529349 \\ \mathrm{H} & -4.659075 & -2.564106 & 2.529349 \\ \mathrm{H} & 0.458140 & 2.640137 & 2.115875 \\ \mathrm{H} & 2.057356 & -1.716829 & 2.115875 \\ \mathrm{H} & -2.515496 & -0.923308 & 2.115875\end{array}$

\section{4h4}

$\begin{array}{lrrr}\mathrm{P} & 1.132304 & 1.533379 & -0.867958 \\ \mathrm{~N} & 1.179436 & 1.539172 & 0.730831 \\ \mathrm{P} & 1.332299 & 0.206912 & 1.602773 \\ \mathrm{~N} & 1.513045 & -1.166345 & 0.802850 \\ \mathrm{P} & 1.476244 & -1.255926 & -0.793767 \\ \mathrm{~N} & 1.319064 & 0.120226 & -1.593691 \\ \mathrm{~S} & 2.417344 & 2.982402 & -1.676093 \\ \mathrm{~S} & 2.802470 & 0.428311 & 3.083873 \\ \mathrm{~S} & 3.079890 & -2.390607 & -1.531939 \\ \mathrm{C} & 1.064126 & 3.918769 & -2.314012 \\ \mathrm{C} & 1.608675 & 0.302707 & 4.377704 \\ \mathrm{C} & 1.996791 & -3.647543 & -2.133547 \\ \mathrm{C} & -0.201400 & 3.382516 & -2.064913 \\ \mathrm{C} & 0.288352 & 0.130576 & 3.955457 \\ \mathrm{C} & 0.637629 & -3.424033 & -1.901383 \\ 0 & -0.274534 & 2.208517 & -1.365601 \\ \mathrm{O} & 0.047024 & 0.076102 & 2.609555 \\ \mathrm{O} & 0.278746 & -2.278322 & -1.244721 \\ \mathrm{C} & 1.188815 & 5.114287 & -3.012854 \\ \mathrm{C} & 1.904614 & 0.355193 & 5.735224 \\ \mathrm{C} & 2.409633 & -4.793107 & -2.804693 \\ \mathrm{C} & 0.035400 & 5.750592 & -3.460946\end{array}$




\begin{tabular}{|c|c|c|c|}
\hline $\mathrm{C}$ & 0.863611 & 0.242970 & 6.651604 \\
\hline $\mathrm{C}$ & 1. 446937 & -5.705225 & -3.225753 \\
\hline $\mathrm{C}$ & -1.229188 & 5. 202548 & -3.204421 \\
\hline $\mathrm{C}$ & -0.457467 & 0.070228 & 6.215 \\
\hline $\mathrm{C}$ & 0.085756 & -5.469539 & -2.9870 \\
\hline $\mathrm{C}$ & -1.357096 & 4. 010911 & -2.4967 \\
\hline $\mathrm{C}$ & -0.755059 & 0.007719 & $4.85^{\prime}$ \\
\hline C & -0.330708 & -4.318879 & -2.32 \\
\hline S & 0.063096 & 7. 231173 & -4.41 \\
\hline S & 1. 098688 & 0.364755 & 8.39 \\
\hline$S$ & 1. 845946 & -7.226254 & -4.02 \\
\hline $\mathrm{C}$ & -1.673333 & 7.514855 & -4.2 \\
\hline $\mathrm{C}$ & -0.531908 & -0.180859 & 8.79 \\
\hline $\mathrm{C}$ & 0.179354 & -7.582995 & -4.479 \\
\hline S & -2.606124 & 6.073438 & -3.86 \\
\hline S & -1.689460 & -0.004514 & 7. 46 \\
\hline S & -1.028078 & -6.723248 & -3.5205 \\
\hline $\mathrm{C}$ & -2.241719 & 8. 717778 & -4.502 \\
\hline $\mathrm{C}$ & -0.872012 & -0.660609 & 10.00 \\
\hline $\mathrm{C}$ & -0.144938 & -8.457687 & -5 \\
\hline S & -3.984955 & 8. 994446 & -4.395 \\
\hline S & -2.513071 & -1.178763 & 10.411 \\
\hline S & -1.815623 & -8.837 & \\
\hline $\mathrm{C}$ & -3.857583 & 10. 736690 & -4.486 \\
\hline $\mathrm{C}$ & -2.057389 & -1.911473 & 11.933 \\
\hline $\mathrm{C}$ & -1.385619 & -9.722524 & -7.3374 \\
\hline $\mathrm{C}$ & -2.653841 & 11. 260477 & -4.7304 \\
\hline $\mathrm{C}$ & -0.799449 & -1.748204 & 12. 3500 \\
\hline $\mathrm{C}$ & -0.089597 & -9.947724 & -7.56657 \\
\hline S & -1.311800 & 10. 157743 & -4.93694 \\
\hline S & 0.280535 & -0.816977 & 11.33701 \\
\hline S & 1. 062371 & -9.336943 & -6.40053 \\
\hline $\mathrm{H}$ & -2.449419 & 12. 318937 & -4.8362 \\
\hline $\mathrm{H}$ & -0.402696 & -2.128066 & 13. 2836 \\
\hline $\mathrm{H}$ & 0.302301 & -10.504266 & -8.4091 \\
\hline $\mathrm{H}$ & -4.769056 & 11. 309493 & -4.3662 \\
\hline $\mathrm{H}$ & -2.826802 & -2.442535 & 12. 4802 \\
\hline $\mathrm{H}$ & -2.195161 & -10.070358 & -7.9675 \\
\hline $\mathrm{H}$ & 2. 167031 & 5.543630 & -3.2016 \\
\hline $\mathrm{H}$ & 2. 928380 & 0.477966 & 6.0724 \\
\hline $\mathrm{H}$ & 3. 462255 & -4.969786 & -2.9987 \\
\hline $\mathrm{H}$ & -2.323870 & 3.572957 & -2.2758 \\
\hline $\mathrm{H}$ & -1.766636 & -0.136678 & 4. 4951 \\
\hline 4 & -1.377700 & -4.108369 & -2.1375 \\
\hline
\end{tabular}




\section{4h5}

\begin{tabular}{|c|c|c|c|}
\hline $\mathrm{P}$ & -0.273144 & 1.598838 & -1.027827 \\
\hline $\mathrm{N}$ & 1. 206787 & 1.001685 & -1.145560 \\
\hline $\mathrm{P}$ & 1. 521207 & -0.562869 & -1.027827 \\
\hline $\mathrm{N}$ & 0.264091 & -1.545951 & -1.145560 \\
\hline P & -1.248062 & -1.035969 & -1.027827 \\
\hline $\mathrm{N}$ & -1.470878 & 0.544266 & -1.145560 \\
\hline $\mathrm{N}$ & -0.499099 & 2.919463 & -2.034706 \\
\hline $\mathrm{N}$ & 2. 777878 & -1.027499 & -2.034706 \\
\hline $\mathrm{N}$ & -2.278780 & -1.891963 & -2.034706 \\
\hline $\mathrm{C}$ & -0.704031 & 4. 096201 & -1.322127 \\
\hline $\mathrm{C}$ & 3. 899429 & -1.438391 & -1.322127 \\
\hline $\mathrm{C}$ & -3.195398 & -2.657809 & -1.322127 \\
\hline $\mathrm{C}$ & -0.658477 & 3.828917 & 0.051362 \\
\hline $\mathrm{C}$ & 3. 645177 & -1.344201 & 0.051 \\
\hline $\mathrm{C}$ & -2.986701 & -2.484716 & 0.051362 \\
\hline 0 & -0.428556 & 2.504308 & 0.335604 \\
\hline 0 & 2. 383072 & -0.881014 & \\
\hline 0 & -1.954516 & -1.623294 & 0.335604 \\
\hline $\mathrm{C}$ & -0.916257 & 5. 385137 & -1.781479 \\
\hline $\mathrm{C}$ & 5. 121794 & -1.899067 & -1.781479 \\
\hline $\mathrm{C}$ & -4.205537 & -3.486070 & -1.781479 \\
\hline $\mathrm{C}$ & -1.091050 & 6. 391794 & -0.827329 \\
\hline $\mathrm{C}$ & 6. 080981 & -2.251020 & -0.827329 \\
\hline $\mathrm{C}$ & -4.989931 & -4.140774 & -0.827329 \\
\hline $\mathrm{C}$ & -1.043198 & 6.112758 & 0.541875 \\
\hline $\mathrm{C}$ & 5.815403 & -2.152943 & 0.541875 \\
\hline $\mathrm{C}$ & -4.772205 & -3.959815 & 0.541875 \\
\hline $\mathrm{C}$ & -0.818484 & 4. 811792 & 1. 002222 \\
\hline $\mathrm{C}$ & 4. 576376 & -1.697068 & 1. 002222 \\
\hline $\mathrm{C}$ & -3.757892 & -3.114724 & 1. 002222 \\
\hline S & -1.436775 & 8. 063434 & -1.269385 \\
\hline S & 7. 701526 & -2.787433 & -1.269385 \\
\hline S & -6.264751 & -5.276000 & -1.269385 \\
\hline $\mathrm{C}$ & -1.203731 & 8. 693054 & 0.362698 \\
\hline $\mathrm{C}$ & 8. 130271 & -3.304066 & 0.362698 \\
\hline $\mathrm{C}$ & -6.926541 & -5.388988 & 0.362698 \\
\hline $\mathrm{S}$ & -1.336025 & 7. 471078 & 1. 628028 \\
\hline S & 7. 138156 & -2.578507 & 1. 628028 \\
\hline S & -5.802131 & -4.892570 & 1. 628028 \\
\hline $\mathrm{C}$ & -0.980623 & 9.997886 & 0.621022 \\
\hline $\mathrm{C}$ & 9. 148735 & -4.149699 & 0.621022 \\
\hline $\mathrm{C}$ & -8.168112 & -5.848187 & 0.6210 \\
\hline
\end{tabular}




$\begin{array}{rrrr}\mathrm{S} & -0.880511 & 11.229351 & -0.645021 \\ \mathrm{~S} & 10.165159 & -4.852131 & -0.645021 \\ \mathrm{~S} & -9.284648 & -6.377220 & -0.645021 \\ \mathrm{C} & -0.280672 & 12.479423 & 0.421932 \\ \mathrm{C} & 10.947833 & -5.996643 & 0.421932 \\ \mathrm{C} & -10.667162 & -6.482780 & 0.421932 \\ \mathrm{C} & -0.233421 & 12.211341 & 1.729222 \\ \mathrm{C} & 10.692042 & -5.903522 & 1.729222 \\ \mathrm{C} & -10.458621 & -6.307819 & 1.729222 \\ \mathrm{~S} & -0.774964 & 10.634427 & 2.258610 \\ \mathrm{~S} & 9.597166 & -4.646075 & 2.258610 \\ \mathrm{~S} & -8.822202 & -5.988352 & 2.258610 \\ \mathrm{H} & -0.491385 & 2.879772 & -3.039375 \\ \mathrm{H} & 2.739648 & -1.014334 & -3.039375 \\ \mathrm{H} & -2.248263 & -1.865438 & -3.039375 \\ \mathrm{H} & -0.941112 & 5.610261 & -2.842520 \\ \mathrm{H} & 5.329185 & -1.990104 & -2.842520 \\ \mathrm{H} & -4.388073 & -3.620158 & -2.842520 \\ \mathrm{H} & 0.000000 & 13.424793 & -0.026246 \\ \mathrm{H} & 11.626212 & -6.712396 & -0.026246 \\ \mathrm{H} & -11.626212 & -6.712396 & -0.026246 \\ \mathrm{H} & 0.091304 & 12.908532 & 2.492049 \\ \mathrm{H} & 11.133465 & -6.533338 & 2.492049 \\ \mathrm{H} & -11.224769 & -6.375194 & 2.492049 \\ \mathrm{H} & -0.766052 & 4.573772 & 2.058420 \\ \mathrm{H} & 4.344029 & -1.623465 & 2.058420 \\ \mathrm{H} & -3.577977 & -2.950307 & 2.058420\end{array}$

\section{4h6}

$\begin{array}{lrrr}\mathrm{P} & 0.000000 & 0.000000 & 0.000000 \\ \mathrm{~N} & 0.000000 & 0.000000 & 1.609823 \\ \mathrm{P} & 1.353131 & 0.000000 & 2.482874 \\ \mathrm{~N} & 2.687906 & 0.437614 & 1.696175 \\ \mathrm{P} & 2.790339 & 0.454114 & 0.088853 \\ \mathrm{~N} & 1.386654 & 0.428290 & -0.698696 \\ \mathrm{~N} & -1.256942 & 0.941669 & -0.594342 \\ \mathrm{~N} & 1.144849 & 0.931371 & 3.863455 \\ \mathrm{~N} & 3.729375 & 1.752681 & -0.413453 \\ \mathrm{C} & -2.219307 & 0.370727 & -1.417079 \\ \mathrm{C} & 1.134610 & 0.327789 & 5.113881 \\ \mathrm{C} & 4.992220 & 1.526263 & -0.945803 \\ \mathrm{C} & -2.113357 & -1.015656 & -1.606781 \\ \mathrm{C} & 1.368889 & -1.055822 & 5.123699 \\ \mathrm{C} & 5.342460 & 0.179965 & -1.130977\end{array}$




\begin{tabular}{|c|c|c|c|}
\hline S & -0.776827 & -1.807476 & -0.766902 \\
\hline S & 1. 637687 & -1.802711 & 3.546219 \\
\hline S & 4. 118737 & -1.004499 & -0.664155 \\
\hline $\mathrm{C}$ & -3.249350 & 1. 084414 & -2.022057 \\
\hline C & 0.925862 & 1. 012068 & 6.307438 \\
\hline 0 & 5. 879207 & 2.537045 & -1.303514 \\
\hline C & -4.161705 & 0.401603 & -2.820556 \\
\hline $\mathrm{C}$ & 0.945057 & 0.299978 & 7. 502753 \\
\hline $\mathrm{C}$ & 7. 116082 & 2. 188287 & -1.837140 \\
\hline $\mathrm{C}$ & -4.056500 & -0.981499 & -3.007629 \\
\hline $\mathrm{C}$ & 1. 180447 & -1.079763 & 7. 511741 \\
\hline $\mathrm{C}$ & 7. 464434 & 0.845220 & -2.021515 \\
\hline $\mathrm{C}$ & -3.031407 & -1.696304 & -2.395908 \\
\hline $\mathrm{C}$ & 1. 398349 & -1.762266 & 6.319047 \\
\hline $\mathrm{C}$ & 6. 574118 & -0.165173 & -1.671941 \\
\hline S & -5.464637 & 1. 208005 & -3.688252 \\
\hline S & 0.630405 & 1. 046417 & 9.066345 \\
\hline S & 8. 342991 & 3. 376639 & -2.266378 \\
\hline $\mathrm{C}$ & -6.277850 & -0.290961 & -4.145792 \\
\hline $\mathrm{C}$ & 1. 136879 & -0.364105 & 9.999935 \\
\hline $\mathrm{C}$ & 9. 373792 & 2. 215297 & -3.106961 \\
\hline S & -5.239904 & -1.716386 & -4.089812 \\
\hline$S$ & 1. 120656 & -1.872993 & 9.086121 \\
\hline$S$ & 9. 084178 & 0.536667 & -2.648228 \\
\hline $\mathrm{C}$ & -7.568189 & -0.334388 & -4.535675 \\
\hline $\mathrm{C}$ & 1. 472582 & -0.297116 & 11.304453 \\
\hline $\mathrm{C}$ & 10. 318948 & 2.582847 & -3.996043 \\
\hline S & -8.599157 & 1. 100294 & -4.623890 \\
\hline S & 1. 456527 & 1. 210387 & 12. 229501 \\
\hline S & 10.630767 & 4. 267927 & -4.433799 \\
\hline $\mathrm{C}$ & -10.078017 & 0.196912 & -4.863363 \\
\hline $\mathrm{C}$ & 2. 275952 & 0.532491 & 13. 618651 \\
\hline $\mathrm{C}$ & 11. 671343 & 3. 872337 & -5.783527 \\
\hline $\mathrm{C}$ & -9.978537 & -1.122760 & -5.040980 \\
\hline $\mathrm{C}$ & 2. 500690 & -0.783725 & 13. 626790 \\
\hline $\mathrm{C}$ & 12. 002681 & 2. 590649 & -5.958152 \\
\hline S & -8.379088 & -1.830638 & -5.017523 \\
\hline S & 1. 956803 & -1.712621 & 12.247958 \\
\hline S & 11. 366192 & 1. 421893 & -4.822844 \\
\hline $\mathrm{H}$ & -1.201541 & 1. 945720 & -0.512955 \\
\hline $\mathrm{H}$ & 0.884547 & 1.901645 & 3. 772289 \\
\hline H & 3. 464338 & 2. 686594 & -0.138760 \\
\hline 11 & -3.342729 & 2. 154715 & -1.865868 \\
\hline $\mathrm{H}$ & 0.759211 & 2. 084839 & 6. 304622 \\
\hline
\end{tabular}




$\begin{array}{rrrr}\mathrm{H} & 5.605491 & 3.579821 & -1.175687 \\ \mathrm{H} & -11.005687 & 0.755833 & -4.883139 \\ \mathrm{H} & 2.539736 & 1.210295 & 14.421356 \\ \mathrm{H} & 12.021218 & 4.691517 & -6.399841 \\ \mathrm{H} & -10.814407 & -1.786673 & -5.225174 \\ \mathrm{H} & 2.972883 & -1.325469 & 14.437322 \\ \mathrm{H} & 12.659607 & 2.222192 & -6.736669 \\ \mathrm{H} & -2.948058 & -2.769670 & -2.531479 \\ \mathrm{H} & 1.589616 & -2.830369 & 6.319990 \\ \mathrm{H} & 6.835375 & -1.208005 & -1.819177\end{array}$


II. Figures not given in the text.
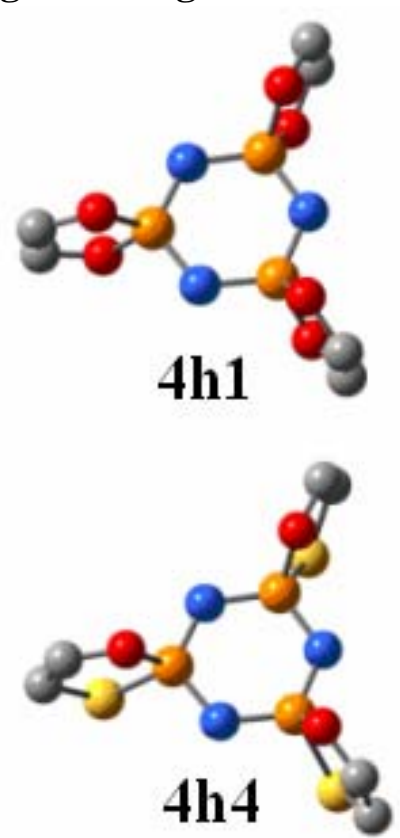
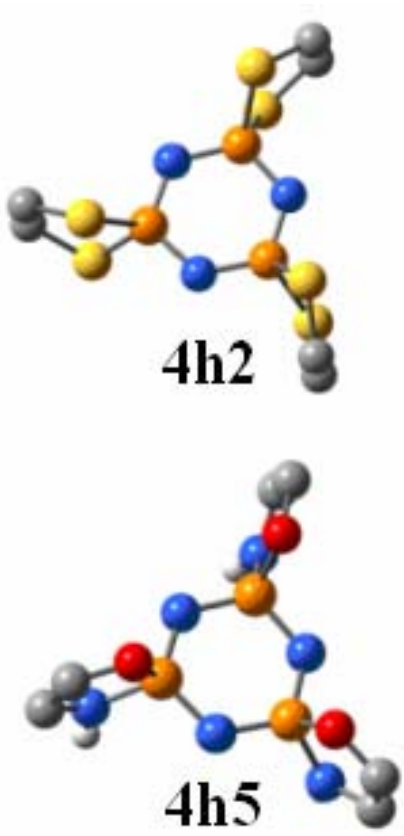
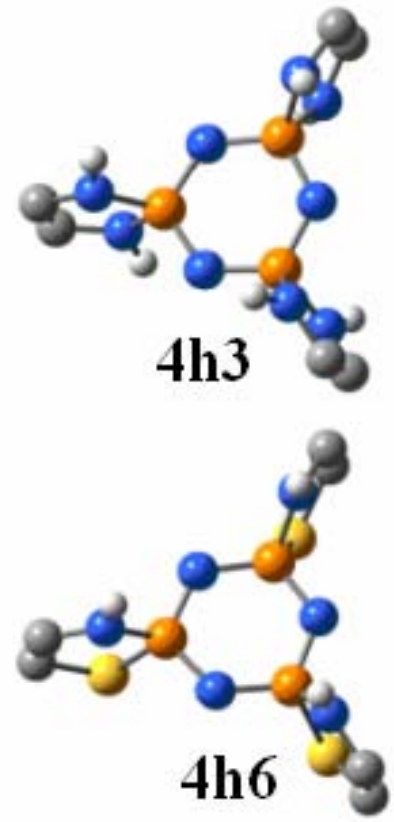

Figure S1. PBE0/6-31G(d,p) optimized structures of representatives of the series of derivatives built up with the $\left[(\mathrm{NP})_{3}\right]$ core $(4)$ and different bridging moieties to the side fragment. Here, we only show the common part of the molecules, and for clarity, orange, gray, red, blue, yellow and while colors are used, respectively for Phosphorus (P), Carbon (C), Oxygen (O), Nitrogen (N), Sulfur (S), and Hydrogen (H).

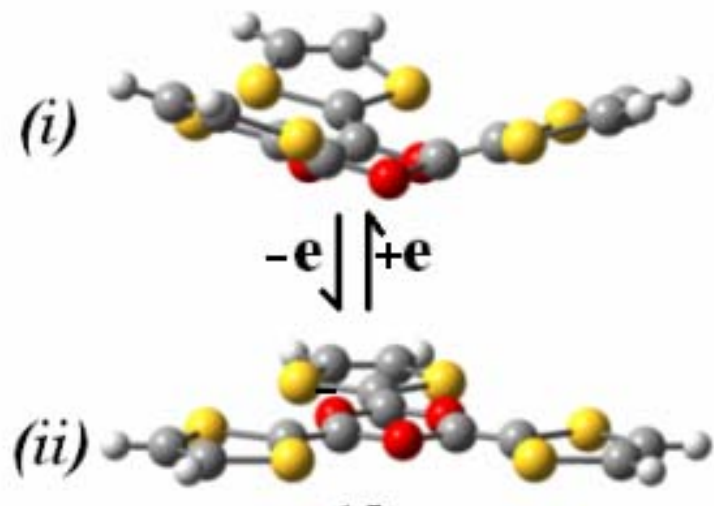

1b
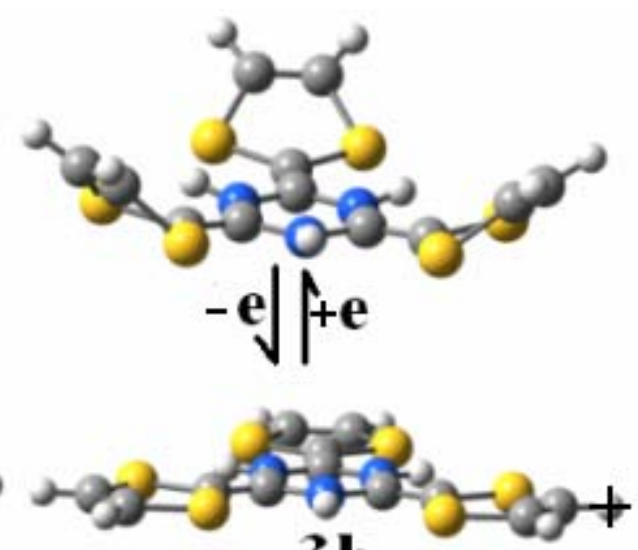

3b

Figure S2. PBE0/6-31G(d,p) optimized structures of the (i) neutral and (ii) oxidized forms for the derivative $\mathbf{1 b}$ and $\mathbf{3 b}$ respectively. 


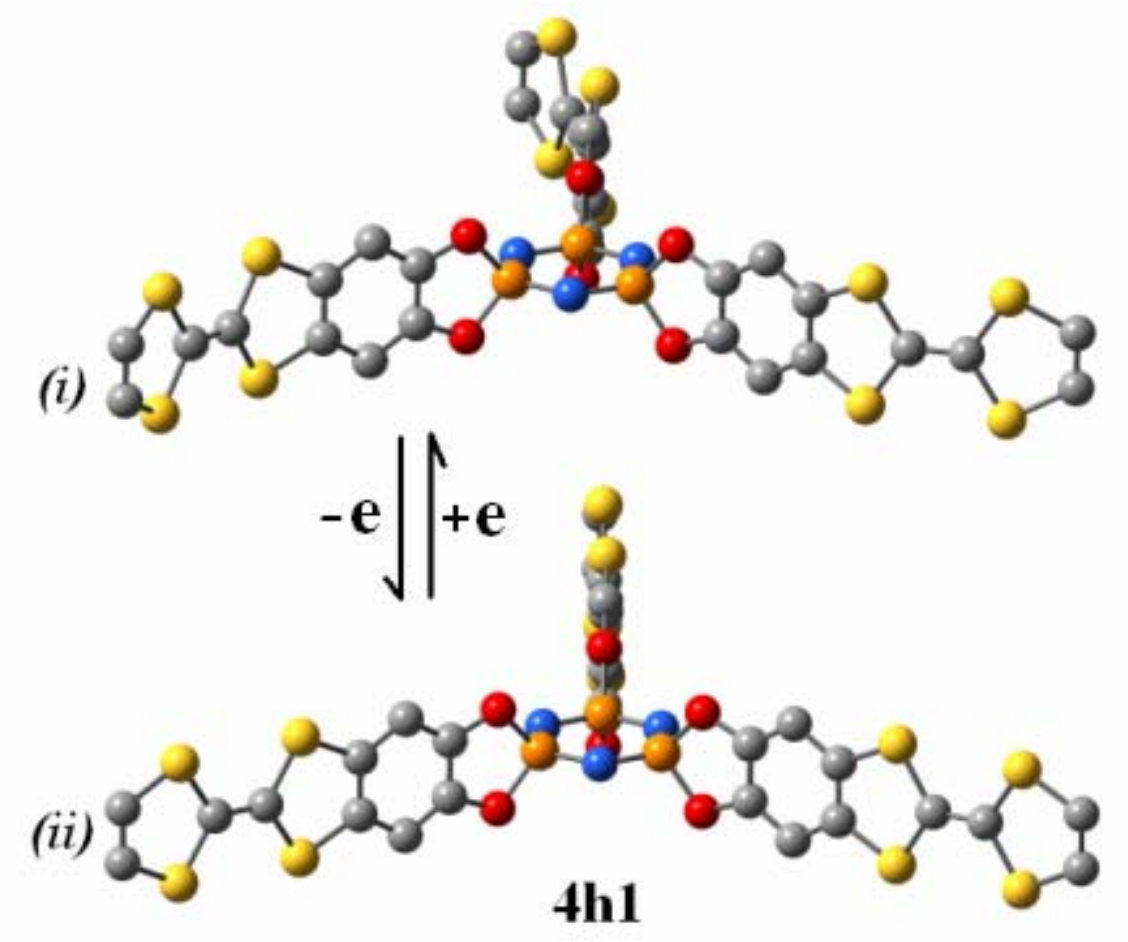

Figure S3. PBE0/6-31G(d,p) optimized structures of the (i) neutral and (ii) oxidized forms for the derivative $\mathbf{4 h 1}$.
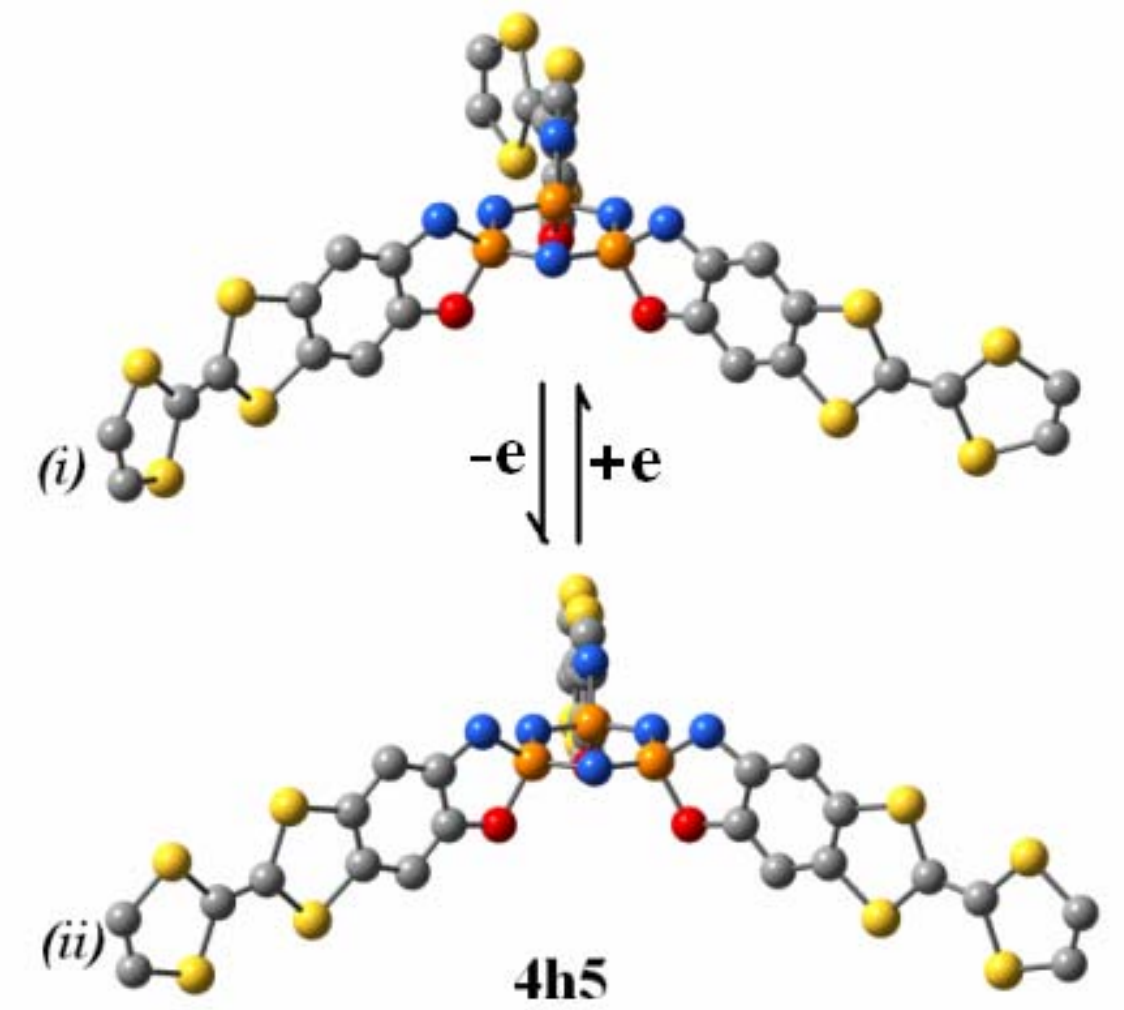

Figure S4. PBE0/6-31G(d,p) optimized structures of the (i) neutral and (ii) oxidized forms for the derivative $\mathbf{4 h} \mathbf{5}$. 


\section{Tables: computational (numerical) results not given in the text.}

Table S1: PBE1PBE/6-31G(d,p) based energetics and spin contamination $\left(S^{2}\right)$ in both relaxed and non relaxed cationic forms). *

\begin{tabular}{|c|c|c|c|c|c|}
\hline Species & $\mathrm{E}_{\mathrm{M}(\mathrm{opt})}$ & $\mathrm{E}_{\mathrm{M}+(\mathrm{opt})}$ & $\mathrm{E}_{\mathrm{M}+(\mathrm{nopt})}$ & $<\mathrm{S}^{2}>_{\mathrm{M}+(\mathrm{opt})}$ & $\left\langle\mathrm{S}^{2}>_{\mathrm{M}+(\text { nopt }}\right.$ \\
\hline TTF & -1822.878751 & -1822.648259 & -1822.643045 & 0.753815 & 0.754207 \\
\hline TMTST & -9984.186447 & -9983.969605 & -9983.956448 & 0.753305 & 0.754285 \\
\hline ET & -3569.704608 & -3569.48139 & -3569.470958 & 0.753609 & 0.754735 \\
\hline BETS & -11573.92531 & -11573.70174 & -11573.68928 & 0.753552 & 0.754752 \\
\hline BO & -2278.082112 & -2277.864649 & -2277.853429 & 0.75410 & 0.754783 \\
\hline TPP & -2331.108194 & -2330.8279122 & -2330.8253942 & 0.75507 & 0.754894 \\
\hline 1a & -5697.6934115 & -5697.4818606 & -5697.469817 & 0.757919 & 0.753376 \\
\hline $1 b$ & -3073.8730288 & -3073.6643263 & -3073.6534316 & 0.759046 & 0.753171 \\
\hline 1c & -15079.9883802 & -15079.7747089 & -15079.7566671 & 0.757604 & 0.752738 \\
\hline 1d & -5694.1036771 & -5693.898077 & -5693.888215 & 0.757057 & 0.753582 \\
\hline 1e & -17700.2361546 & -17700.0245513 & -17700.0092669 & 0.755836 & 0.753277 \\
\hline 1f & -3756.6572403 & -3756.4582062 & -3756.4489007 & 0.750038 & 0.753009 \\
\hline $2 \mathbf{b}$ & -4042.5890625 & -4042.3632951 & -4042.3498824 & 0.75033 & 0.769771 \\
\hline $3 \mathbf{b}$ & -3014.3641357 & -3014.1648797 & -3014.1501616 & 0.753407 & 0.754739 \\
\hline 4b & -3921.9251831 & -3921.7450726 & -3921.720052 & 0.757778 & 0.798168 \\
\hline 4g1 & -10188.0550366 & -10187.845232 & -10187.8399021 & 0.751760 & 0.751838 \\
\hline 4h1 & -7564.2329929 & -7564.0257759 & -7564.0202568 & 0.751704 & 0.751827 \\
\hline $4 \mathrm{i1}$ & -31576.4679953 & -31576.2585478 & -31576.2509875 & 0.751596 & 0.751858 \\
\hline $4 j 1$ & -10184.4656028 & -10184.2579948 & -10184.2533151 & 0.751645 & 0.751734 \\
\hline $4 \mathbf{k} 1$ & -34196.7191519 & -34196.5087302 & -34196.5046886 & 0.751595 & 0.751669 \\
\hline 411 & -8247.0173342 & -8246.8166159 & -8246.8114151 & 0.751689 & 0.751841 \\
\hline $4 h 2$ & -9501.5415218 & -9501.3326297 & -9501.3272489 & 0.751780 & 0.751897 \\
\hline $4 i 2$ & -33513.77592 & -33513.5648879 & -33513.5593289 & 0.751641 & 0.751956 \\
\hline $4 j 2$ & -12121.7738521 & -12121.5648835 & -12121.5605409 & 0.751710 & 0.751743 \\
\hline $4 \mathbf{k} 2$ & -36134.0267516 & na & -36133.811564 & na & 0.751472 \\
\hline 412 & -10184.325966 & -10184.1238803 & -10184.1182939 & 0.751806 & 0.751934 \\
\hline $4 h 3$ & -7445.1205496 & -7444.9240161 & -7444.9183537 & 0.751951 & 0.751979 \\
\hline $4 i 3$ & -31457.355419 & -31457.1561398 & -31457.1498214 & 0.751739 & 0.751790 \\
\hline $4 j 3$ & -10065.3541289 & -10065.1552951 & -10065.1509579 & 0.751849 & 0.751876 \\
\hline $4 \mathbf{k} 3$ & -34077.6075006 & -34077.4058546 & -34077.4019601 & 0.751804 & 0.751782 \\
\hline 413 & -8127.9052649 & -8127.7132223 & -8127.7075842 & 0.751610 & 0.751761 \\
\hline $4 h 4$ & -8532.8918832 & -8532.6844549 & -8532.6789438 & 0.751739 & 0.751865 \\
\hline $4 i 4$ & -32545.1266705 & 32544.9169708 & -32544.911012 & 0.751615 & 0.751732 \\
\hline $4 j 4$ & -11153.124393 & -11152.9164588 & -11152.9121853 & 0.751665 & 0.751721 \\
\hline $4 \mathbf{k} 4$ & -35165.377683 & na & -35165.1627016 & na & 0.751655 \\
\hline 414 & -9215.6762042 & -9215.475291 & -9215.4698422 & 0.751708 & 0.751881 \\
\hline $4 h 5$ & -7504.678501 & -7504.4776688 & -7504.4721963 & 0.751776 & 0.751865 \\
\hline $4 \mathrm{i} 5$ & -31516.9134718 & -31516.7102565 & -31516.7039579 & 0.751632 & 0.751723 \\
\hline
\end{tabular}




\begin{tabular}{clllcc}
\hline $\mathbf{4 j 5}$ & -10124.9116632 & -10124.7093883 & -10124.7048422 & 0.751697 & 0.751758 \\
$\mathbf{4 k 5}$ & -34137.1651139 & -34136.9602278 & -34136.955852 & 0.751666 & 0.751692 \\
$\mathbf{4 l 5}$ & -8187.4630354 & -8187.2677588 & -8187.2620571 & 0.751593 & 0.751737 \\
$\mathbf{4 h 6}$ & -8473.3347809 & -8473.1320741 & -8473.1265197 & 0.751819 & 0.751895 \\
$\mathbf{4 i 6}$ & -32485.5697747 & -32485.3644446 & -32485.3587346 & 0.751819 & 0.751825 \\
$\mathbf{4 j 6}$ & -11093.5679419 & -11093.3636685 & -11093.3593185 & 0.75171 & 0.751779 \\
$\mathbf{4 k 6}$ & -35105.8205948 & na & -35105.6097574 & na & 0.751687 \\
$\mathbf{4 l 6}$ & -9156.1192355 & -9155.9221838 & -9155.9166262 & 0.751636 & 0.751758 \\
\hline
\end{tabular}

* na : calculation not achieved; $\mathrm{M}(\mathrm{opt})=$ optimized geometry of the neutral form; $\mathrm{M}^{+}$(opt)

= optimized geometry of the oxidized form; $\mathrm{M}^{+}$(nopt) = oxidized form in the optimized geometry of the neutral form. 
Table S2. PBE1/6-31G+(d,p) computed energetics.

\begin{tabular}{|c|c|c|c|}
\hline Species & $\mathbf{M}^{0 a}$ & $\mathrm{M}^{+}(\mathrm{opt})^{b}$ & $\mathrm{M}^{+}(\text {nopt })^{c}$ \\
\hline TTF & -1822.8787527 & -1822.6482638 & -1822.6430502 \\
\hline TMTST & -9984.1864487 & -9983.9696523 & -9983.956453 \\
\hline ET & -3569.7046102 & -3569.4813935 & -3569.4709626 \\
\hline BETS & -11573.9253187 & -11573.7017473 & -11573.6892875 \\
\hline BO & -2278.0821363 & -2277.8647024 & -2277.8534314 \\
\hline TPP & -2331.1487461 & -2330.8627979 & -2330.8599095 \\
\hline 1a & -5697.7208884 & -5697.5039051 & -5697.4932076 \\
\hline $1 b$ & -3073.8949671 & -3073.6797374 & -3073.6697015 \\
\hline 1c & -15080.2033914 & -15080.0034594 & -15079.9652352 \\
\hline 1d & -5694.1306485 & -5693.9192875 & -5693.9109433 \\
\hline 1e & -17700.4757245 & -17700.2648615 & -17700.2442529 \\
\hline 1f & -3756.6992194 & -3756.4932068 & -3756.4845976 \\
\hline $2 \mathbf{b}$ & -4042.6076222 & -4042.3776348 & -4042.3641077 \\
\hline $\mathbf{3 b}$ & -3014.386154 & -3014.1815099 & -3014.1669742 \\
\hline $4 b$ & -3921.9511302 & -3921.7653649 & -3921.740925 \\
\hline 4g1 & -10188.1170904 & -10187.9029838 & -10187.8981244 \\
\hline $4 g 1$ & -7564.2894324 & -7564.0768748 & -7564.0720418 \\
\hline $4 \mathrm{i1}$ & -31576.9345972 & -31576.7245286 & -31576.7133657 \\
\hline $4 j 1$ & -10184.5275859 & -10184.315423 & -10184.3114467 \\
\hline $4 \mathbf{k} 1$ & -34197.2081241 & & \\
\hline 411 & -8247.0940831 & -8246.8878033 & -8246.8827753 \\
\hline $4 h 1$ & -7564.2894324 & -7564.0768748 & -7564.0720418 \\
\hline $4 \mathrm{~h} 2$ & -9501.5941443 & -9501.3802629 & -9501.375922 \\
\hline $4 \mathrm{~h} 3$ & -7445.1750282 & -7444.9730599 & -7444.9676926 \\
\hline $4 \mathrm{~h} 4$ & -8532.9478163 & -8532.7358935 & -8532.7307604 \\
\hline $4 \mathrm{~h} 5$ & -7504.7341352 & -7504.5279206 & -7504.52290 \\
\hline $4 \mathrm{hj} 6$ & -8473.3893118 & -8473.1817846 & -8473.1766175 \\
\hline
\end{tabular}

${ }^{a}$ Optimized structure of the neutral form; ${ }^{b}$ Optimized structure of the oxidized form; Oxidized form in the optimized structure of the neutral form. 
Table S3: Optimized structure of TPP as function of theoretical method *

\begin{tabular}{|c|c|c|c|c|c|c|}
\hline Parameter & B3LYP & B3P86 & BP86 & PBE0 & $\mathrm{HF}$ & Exptl. $^{\mathrm{b}}$ \\
\hline \multicolumn{7}{|c|}{ Bond length $(\AA)$} \\
\hline $\mathrm{P}-\mathrm{N}$ & 1.597 & 1.592 & 1.613 & 1.591 & 1.573 & 1.575 \\
\hline $\mathrm{P}-\mathrm{O}$ & 1.645 & 1.638 & 1.664 & 1.636 & 1.610 & 1.613 \\
\hline $\mathrm{O}-\mathrm{C}$ & 1.388 & 1.380 & 1.396 & 1.378 & 1.370 & 1.393 \\
\hline $\mathrm{C}_{1}-\mathrm{C}_{1}$, & 1.394 & 1.391 & 1.404 & 1.391 & 1.380 & 1.373 \\
\hline $\mathrm{C}_{1}-\mathrm{C}_{2}$ & 1.382 & 1.379 & 1.391 & 1.379 & 1.368 & 1.357 \\
\hline $\mathrm{C}_{2}-\mathrm{C}_{3}$ & 1.403 & 1.399 & 1.410 & 1.399 & 1. 395 & 1.387 \\
\hline $\mathrm{C}_{3}-\mathrm{C}_{3}$, & 1.396 & 1.393 & 1.405 & 1.393 & 1.383 & 1.360 \\
\hline \multicolumn{7}{|c|}{ Bond Angle $\left({ }^{\circ}\right)$} \\
\hline P-N-P & 122.736 & 122.630 & 121.542 & 122.554 & 124.428 & 121.300 \\
\hline N-P-N & 117.264 & 117.370 & 118.458 & 117.445 & 115.572 & 118.633 \\
\hline N-P-O & & 110.384 & 110.025 & 110.343 & & 109.833 \\
\hline O-P-O & 95.807 & 95.932 & 96.141 & 96.004 & 95.404 & 96.667 \\
\hline $\mathrm{P}-\mathrm{O}-\mathrm{C}_{1}$ & 109.920 & 109.847 & 109.338 & 109.789 & 110.868 & 108.000 \\
\hline $\mathrm{O}-\mathrm{C}_{1}-\mathrm{C}_{1}$, & 112.176 & 112.190 & 112.593 & 112.211 & 111.430 & 111.83 \\
\hline $\mathrm{O}-\mathrm{C}_{1}-\mathrm{C}_{2}$ & & 126.035 & 125.666 & 126.020 & & 126.167 \\
\hline $\mathrm{C}_{1},-\mathrm{C}_{1}-\mathrm{C}_{2}$ & 121.781 & 121.775 & 121.741 & 121.770 & 121.916 & 121.000 \\
\hline $\mathrm{C}_{1}-\mathrm{C}_{2}-\mathrm{C}_{3}$ & 116.916 & 117.004 & 117.027 & 117.012 & 116.916 & 116.333 \\
\hline
\end{tabular}

* See Scheme 2 for the labeling scheme. 6-31G(d,p) basis set was used in all cases; ${ }^{b}$

Data from Ref. 34 
Table S4: Frontier Molecular Orbital energy ( $E_{\text {HOMO }}$ and $\left.E_{\mathrm{LUMO}}\right)$ and HOMO-LUMO gap $(\Delta E)$ as function of computational method.

\begin{tabular}{|c|c|c|c|c|c|c|}
\hline \multirow[b]{2}{*}{ Species } & \multicolumn{3}{|c|}{ PBE0/6-31G**//PBE0/6-31G** } & \multicolumn{3}{|c|}{$\mathrm{HF} / 6-31 \mathrm{G}^{* *} / / \mathrm{PBE} 0 / 6-31 \mathrm{G}^{* *}$} \\
\hline & $E_{\text {НОмо }}$ & $E_{\text {LUMO }}$ & $\Delta E$ & $E_{\text {НОМО }}$ & $E_{\text {LUMO }}$ & $\Delta E$ \\
\hline TTF & -4.67492 & -0.74967 & 3. 925244 & -0.24473 & 0.11430 & 0.35903 \\
\hline TMTSF & -4.70077 & -0.97254 & 3. 728234 & -0.24442 & 0.09356 & 0.33798 \\
\hline ET & -4.97397 & -0.87784 & 4. 096132 & -0.2586 & 0.10679 & 0.36539 \\
\hline BETS & -5.05697 & -1.26778 & 3. 789187 & -0.25921 & 0.08195 & 0.34116 \\
\hline BO & -4.63356 & -0.90451 & 3. 72905 & -0.25083 & 0.10561 & 0.35644 \\
\hline TPP & -6.59822 & -0.15429 & 6. 443932 & -0.32292 & 0.13012 & 0.45304 \\
\hline 1a & -5.05942 & -0.73063 & 4. 32879 & -0.26809 & 0.10789 & 0.37598 \\
\hline $1 \mathbf{b}$ & -4.74621 & -0.14041 & 4. 605802 & -0.25505 & 0.14059 & 0.39564 \\
\hline 1c & -5.08744 & -0.67185 & 4. 415594 & -0.26745 & 0.11076 & 0.37821 \\
\hline 1d & -4.84036 & -0.39484 & 4. 445526 & -0.25938 & 0.12749 & 0.38687 \\
\hline $1 e$ & -5.12255 & -0.77552 & 4. 347021 & -0.26549 & 0.10384 & 0.36933 \\
\hline 1f & -4.55682 & -0.41252 & 4. 144296 & -0.25324 & 0.12843 & 0.38167 \\
\hline $1 b$ & -4.74621 & -0.14041 & 4. 605802 & -0.25505 & 0.14059 & 0.39564 \\
\hline $2 \mathbf{b}$ & -5.31928 & -0.563 & 4. 756281 & -0.27234 & 0.11416 & 0.3865 \\
\hline $3 \mathbf{b}$ & -4.63056 & -0.24191 & 4. 388655 & -0.24541 & 0.13388 & 0.37929 \\
\hline $4 b$ & -4.45832 & -1.77282 & 2. 685493 & -0.22855 & 0.0441 & 0.27265 \\
\hline $4 g 1$ & -5.17996 & -1.03703 & 4. 142936 & -0.26343 & 0.09695 & 0.36038 \\
\hline $4 h 1$ & -5.05152 & -0.94777 & 4. 103751 & -0.25806 & 0.1042 & 0.36226 \\
\hline $4 \mathrm{i1}$ & -5.14731 & -1.31186 & 3.835447 & -0.25972 & 0.08143 & 0.34115 \\
\hline $4 j 1$ & -5.11139 & -1.01199 & 4. 099397 & -0.26189 & 0.10124 & 0.36313 \\
\hline $4 \mathbf{k} 1$ & -5.18568 & -1.38152 & 3. 804154 & -0.2632 & 0.07761 & 0.34081 \\
\hline 411 & -4.9103 & -1.03186 & 3. 878441 & -0.25786 & 0.1003 & 0.35816 \\
\hline $4 \mathrm{~h} 2$ & -5.10078 & -1.51078 & 3.59 & -0.25905 & 0.07623 & 0.33528 \\
\hline $4 i 2$ & -5.18949 & -1.5252 & 3. 664287 & -0.26061 & 0.07408 & 0.33469 \\
\hline $4 j 2$ & -5.15275 & -1.53336 & 3. 619388 & -0.2628 & 0.07477 & 0.33757 \\
\hline $4 \mathrm{k} 2$ & -5.21262 & -1.55812 & 3.654491 & -0.26362 & 0.07190 & 0.33552 \\
\hline 412 & -4.95574 & -1.49418 & 3. 461562 & -0.25899 & 0.07574 & 0.33473 \\
\hline 4h3 & -4.77397 & -0.72464 & 4. 049328 & -0.24584 & 0.11311 & 0.35895 \\
\hline $4 i 3$ & -4.88526 & -1.09907 & 3. 786194 & -0.248 & 0.08957 & 0.33757 \\
\hline $4 j 3$ & -4.8654 & -0.80845 & 4. 056948 & -0.2506 & 0.10905 & 0.35965 \\
\hline $4 k 3$ & -4.94486 & -1.19812 & 3. 746738 & -0.25171 & 0.08464 & 0.33635 \\
\hline 413 & -4.68608 & -0.82641 & 3. 859665 & -0.24695 & 0.10839 & 0.35534 \\
\hline $4 h 4$ & -5.06459 & -0.97961 & 4. 084975 & -0.25795 & 0.10137 & 0.35932 \\
\hline $4 i 4$ & -5.15602 & -1.32628 & 3. 829732 & -0.25956 & 0.08093 & 0.34049 \\
\hline $4 j 4$ & -5.1220 & -1.03811 & 4. 083887 & -0.26176 & 0.09891 & 0.36067 \\
\hline $4 k 4$ & -5.18595 & -1.40166 & 3.784289 & -0.26264 & 0.07689 & 0.33953 \\
\hline 414 & -4.92662 & -1.05118 & 3. 875448 & -0.25801 & 0.09878 & 0.35679 \\
\hline $4 h 5$ & -4.88064 & -0.80301 & 4. 077628 & -0.25028 & 0.11036 & 0.36064 \\
\hline
\end{tabular}




\begin{tabular}{ccccccc}
\hline $\mathbf{4 i 5}$ & -4.98622 & -1.17798 & 3.808235 & -0.25279 & 0.08657 & 0.33936 \\
$\mathbf{4 j 5}$ & -4.96363 & -0.87866 & 4.084975 & -0.25544 & 0.10642 & 0.36186 \\
$\mathbf{4 k 5}$ & -5.04554 & -1.25907 & 3.786466 & -0.25684 & 0.08228 & 0.33912 \\
$\mathbf{4 l 5}$ & -4.76961 & -0.89716 & 3.872454 & -0.2514 & 0.10565 & 0.35705 \\
$\mathbf{4 h 6}$ & -4.94486 & -0.88981 & 4.055043 & -0.25273 & 0.10536 & 0.35809 \\
$\mathbf{4 i 6}$ & -5.04309 & -1.24492 & 3.798167 & -0.25447 & 0.08392 & 0.33839 \\
$\mathbf{4 j 6}$ & -5.01724 & -0.95539 & 4.061846 & -0.25699 & 0.1023 & 0.35929 \\
$\mathbf{4 k 6}$ & -5.10295 & -1.32383 & 3.779119 & -0.25856 & 0.07973 & 0.33829 \\
$\mathbf{4 1 6}$ & -4.82540 & -0.97226 & 3.853134 & -0.25312 & 0.10157 & 0.35469 \\
\hline
\end{tabular}

